\title{
NONLINEAR INSTABILITY IN DISSIPATIVE FINITE DIFFERENCE SCHEMES*
}

\author{
ANDREW STUART $\dagger$
}

\begin{abstract}
A unified analysis of reaction-diffusion equations and their finite difference representations is presented. The parallel treatment of the two problems shows clearly when and why the finite difference approximations break down. The approach used provides a general framework for the analysis and interpretation of numerical instability in approximations of dissipative nonlinear partial differential equations.

Continuous and discrete problems are studied from the perspective of bifurcation theory, and numerical instability is shown to be associated with the bifurcation of periodic orbits in discrete systems. An asymptotic approach, due to Newell (SIAM J. Appl. Math., 33 (1977), 133-160), is used to investigate the instability phenomenon further. In particular, equations are derived that describe the interaction of the dynamics of the partial differential equation with the artefacts of the discretisation.
\end{abstract}

Key words. continuous and discrete problems, dissipation and nonlinearity, bifurcation and instability

AMS(MOS) subject classifications. 35A40, 35K57, 65M10

1. Introduction. In this paper we analyse two problems: a scalar reactiondiffusion equation and its finite difference analogue. The problems appear below.

Problem (P). Find $u(x, t)$ satisfying

$$
\begin{aligned}
& u_{t}=u_{x x}+\lambda f(u) \\
& u(0, t)=u(1, t)=0, \quad u(x, 0)=u_{0}(x)
\end{aligned}
$$

Problem (PD). Find $u_{j}^{n}$ satisfying

$$
\begin{aligned}
& u_{j}^{n+1}-u_{j}^{n}=r \delta_{x}^{2}\left[\theta u_{j}^{n+1}+(1-\theta) u_{j}^{n}\right]+\lambda \Delta t\left[\theta f\left(u_{j}^{n+1}\right)+(1-\theta) f\left(u_{j}^{n}\right)\right], \\
& u_{0}^{n}=u_{J}^{n}=0, \quad u_{j}^{0}=u_{0}(j \Delta x)
\end{aligned}
$$

${ }_{j}$ for $j=1, \cdots, J-1$. Here $\delta_{x}^{2} U_{j}=U_{j+1}-2 U_{j}+U_{j-1}, J \Delta x=1, r=\Delta t / \Delta x^{2}$, and $0 \leqq \theta \leqq 1$.

Thus $u_{j}^{n}$ approximates $u(j \Delta x, n \Delta t)$. By studying the two problems in parallel we develop an understanding of the approximation of partial differential equations in which a dissipative mechanism is balanced by nonlinearity. The two fundamental questions we ask about the approximation of $(\mathrm{P})$ by $(\mathrm{PD})$ are:

Question 1. What qualitative features of the partial differential equation (P) cannot be adequately represented by the discretisation (PD)?

Question 2. What qualitative features of the discretisation (PD) are not present in the partial differential equation $(\mathrm{P})$ ?

We analyse the behaviour of $(\mathrm{P})$ by means of the elements of local bifurcation, linear stability, and weakly nonlinear stability theories. Such local theories cannot completely explain the global behaviour of the reaction-diffusion equation. Nonetheless, analyses based on these local theories form the cornerstone of many global

* Received by the editors December 7, 1987; accepted for publication (in revised form) December $30,1988$.

†Department of Mathematics, Massachusetts Institute of Technology, Cambridge, Massachusetts, 02139. Present address, School of Mathematics, University of Bath, Claverton Down, Bath BA2 7AY, United Kingdom. 
theories, and the local results themselves yield direct insight into the behaviour of (P) in the vicinity of hyperbolic stationary points.

We apply similar local analyses to (PD). The framework within which we study the finite difference equations is that of practical numerical stability (P-stability), rather than convergence. That is, we examine the behaviour of $(\mathrm{P})$ for fixed, but small, mesh-spacings. As for the continuous case, the local analyses do not explain the behaviour of $(\mathrm{P})$ in its entirety; they do, however, illuminate the answers to Questions 1 and 2.

The pioneering paper in the analysis of numerical instability in discrete parabolic problems, by means of the methods from bifurcation and stability theory, is that of Newell [38]. By generalising the method of multiple scales to difference equations, Newell extended weakly nonlinear analyses, developed in the context of hydrodynamic stability theory [53], [56], to discrete equations. He used this asymptotic method to analyse numerical instability in representative dissipative and dispersive discretisations.

The study of the qualitative behaviour of discretisations of differential equations has benefited considerably in recent years from the use of concepts and analytical tools originally developed for continuous problems. This has led to a deeper understanding of the approximation process, and at the same time to simple interpretations of discretisation error in terms readily understood by those applying numerical methods to real problems. For example, a wide class of numerical instabilities can be interpreted as spurious triad wave interactions [10] generated by discretisation. The phenomenon of sideband instability in numerical methods was observed experimentally in [6] and has been analysed further in [48], where it is described in terms of the Benjamin-Feir instability from wave motion [2]. Also relevant in this context is the work of Moore [37], who identified a spurious triad interaction in a numerical model for wave propagation and analysed it by means of the Stokes expansion and multiple scaling arguments. This approach is applicable to a wide class of dispersive difference schemes and has been used to analyse discretisations of the Korteweg-deVries (KdV) equation (Herbst and Cloot [22]) and the inviscid Burger's equation (Cloot and Herbst [9]). Moore's analysis hinges on a comparison of the discrete dispersion relation with the continuous problem. The study of dispersion relations is useful both as a diagnostic and as the basis for analysis. For a survey of such results for linear problems see [54], [55].

The majority of the analyses described here are scattered throughout a broad body of literature in applied mathematics and numerical analysis. Thus it seems appropriate to present the study of both the reaction-diffusion equation and its discretisation in a unified way. The new work presented in this paper is an analytical description of the nonlinear interaction of a high wavenumber mode, which is a product of the discretisation, and a low wavenumber mode present in the governing differential equation. This is of interest since it shows how nonlinear numerical instability can be triggered by a mechanism inherent in the underlying partial differential equation-something that cannot happen in linear problems. The phenomenon of high/low wavenumber interaction has been identified in problems similar to $(\mathrm{P})$, but with homogeneous Neumann or periodic boundary conditions, using a closed subsystem generated by aliasing as the basis for analysis (see [51]).

Aliasing [50, p. 463] has formed the basis for many studies of nonlinear numerical instability, the foundational paper being [40]. However, the approach suffers from a major disadvantage: it generally applies only to the analysis of the interaction of a (relatively small) number of high wavenumber modes (the example in [51] is atypical 
in this respect since the special structure of the problem allows the inclusion of a nontrivial low wavenumber mode). Thus the instabilites predicted by these analyses [6], [9], [13], [16], [22], [48] are based on the assumption that initially a significant amount of energy is found in the high wavenumbers. For problems with smooth initial data this is not the case, and the instability predicted via the aliasing analysis may or may not appear in practice.

In Burgers' equation there is a rapid cascade of energy to high wavenumbers through the formation of shocks, and the aliasing analysis of Fornberg [16] is relevant to a large class of problems with smooth initial data. However, for many other problems there remains the open question of how the energy distributes itself among Fourier modes as the timestepping proceeds. Nonetheless, Moore [37] shows that considerable insight into this question can be obtained by using asymptotic analysis to identify the interactions of the Fourier modes that dominate. Moore's method works well for dispersive equations; in these problems wave interactions determine the departure from a given initial state, and multiple scales arguments can be used to study the interactions. For dissipative problems like (P) and (PD), multiple scaling methods also form the basis for the analysis of departures from a given state. We use the method of Newell [38] to show that there is a direct interaction between the low and high wavenumber modes in (PD). This has an important consequence, since it means that the time scale on which the potentially destabilising high wavenumber modes are stimulated is not governed by the usual cascade of energy through higher and higher wavenumber modes, but by the direct transfer between low and high wavenumbers.

The interaction of the two modes is associated with the interaction of the dynamics of the partial differential equation (represented by the low wavenumber mode) and the artefacts of the discretisation (represented by the high wavenumber mode). The crucial parameter in (P) is $\lambda$; the crucial parameters in (PD) are $\lambda$ and $r$. These parameters define the structure of the bifurcation diagram for (P) and (PD). The parameter $\lambda$ controls the bifurcation of steady solutions in both $(\mathrm{P})$ and (PD). Determining the set of critical values of $\lambda$ for (P) and (PD) forms the basis of the answer to Question 1. The parameter $r$ controls the bifurcation of time-periodic orbits in (PD), a phenomenon that cannot occur in (P). Determining the smallest critical value of $r$ for (PD) forms the basis of the answer to Question 2. In general we will consider problems of arbitrary, but fixed, dimension - that is, $J$, and hence $\Delta x$, will be fixed. Thus the critical value for $r$ implicitly determines a critical value for $\Delta t$.

Let $\lambda_{c}$ denote the smallest critical value of $\lambda$ and $r_{c}$ the smallest critical value of $r$. The following table summarises the previous local studies of (P) and (PD); numerical methods valid for $\lambda \approx \lambda_{c}$ are described in [29]. An asterisk denotes a piece of work for which a standard text or review is cited. Note that $r$ plays no part in $(\mathrm{P})$.

$\begin{array}{lccc} & \lambda \approx \lambda_{c} & r \approx r_{c} & r \approx r_{c} \text { and } \lambda \approx \lambda_{c} \\ \text { (P) Linear } & {[49]^{*}} & - & - \\ \text { (P) Nonlinear } & {[35]} & - & - \\ \text { (PD) Linear } & \text { This work* } & {[41]^{*}} & \text { Thiswork } \\ \text { (PD) Nonlinear } & {[58]} & {[38]} & \text { This work }\end{array}$

This paper contains two main components: in $\$ 2$ we describe known results about $(\mathrm{P})$, and in $\S 3$ we derive analogous results for (PD). Section 2 is divided into four subsections concerned with, respectively, the properties of the eigenvalues of the linearisation of the partial differential equation, a local bifurcation analysis for the steady solutions, a weakly nonlinear analysis of the time-dependent problem, and 
bifurcation from a parameter value at infinity. Section 3 contains analyses similar to those in $\$ 2$ but is longer, since the range of behaviour of the discretisation is far richer than that of the underlying partial differential equation. Section 4 contains a summary and various concluding remarks.

We emphasise again that all the work contained in this paper is of a purely local nature. There is a great deal of current interest in questions concerning the global nonlinear stability of numerical approximations to partial differential equations. Most of that research is concerned with defining numerical stability for nonlinear problems in such a way that the Lax equivalence theorem [41], which concerns the relationships between consistency, stability, and convergence in linear problems, can be generalised [11], [30], [31]. The results in [30] provide a unifying framework for the analysis of discretisations in the limit as the mesh-spacing shrinks to zero. However, here we take the view that, in many practical large-scale computations, it is at least as important to understand how the qualitative behaviour of the discretisations compares with that of the underlying differential equation as it is to develop a convergence proof, since the limit "mesh-spacing shrinks to zero" is unattainable in practice. In particular, the asymptotic behaviour of discretisations of time-dependent problems for fixed values of the mesh-spacing is of fundamental importance in many applications. This standpoint is taken, for example, by Sanz-Serna [44], who studies the computation of periodic orbits by means of symplectic difference schemes (which mimic the areapreserving properties in Hamiltonian systems), and by Iserles [24], who analyses the asymptotics of Runge-Kutta and multistep methods applied to scalar ordinary differential equations.

We hope that, by analysing in detail the local behaviour of a scalar reactiondiffusion equation and its discretisation, we can shed light on the approximation process for the wide class of equations in which a dissipative mechanism is balanced by nonlinearity. Our analysis concerns the behaviour of the partial differential equation and its discretisation near a trivial solution (zero). The ideas, however, apply more generally in the neighbourhood of any steady solution. We show this in $\S 3.3$ by means of secondary bifurcation analysis, and also in [52], where numerical methods for a generalisation of (P) (to allow for convection) are studied using singularity theory.

Problem (P) has a long history and finds application in the modeling of many biological and chemical phenomena. In the case $f(u) \propto e^{u}$ it arises in the theory of combustion, where $u$ represents departure of the temperature from an initial profile. Blowup can occur; this has an interpretation in ignition theory [25]. For $f(u)=u(1-u)$ the equation arises in population genetics, where $u$ is the frequency of a favourable gene; various generalisations of this nonlinearity are also relevant to this application [14]. Similar nonlinearities also arise in studies of the spatial patterning of the spruce budworm [32]. We will study particular classes of nonlinearity which make the exposition simpler without changing the central ideas. Arbitrary nonlinearities are analysed in [52]. Problem (P) is also important, since it represents the enormous class of real-world problems in which a dissipative mechanism competes with nonlinear effects.

2. The continuous problem. In this section we describe the behaviour of $(\mathrm{P})$. We consider two classes of nonlinearity $f(u)$, namely

(i) $f(u): f(0)=0, f^{\prime}(0)=1, f^{\prime \prime}(0)=0, f^{\prime \prime \prime}(0) \neq 0$;

(ii) $f(u): f(0)=f^{\prime}(0)=0, f^{\prime \prime}(0) \neq 0$.

In general it is important to define appropriate function spaces for $(\mathrm{P})$. However, since our analysis is a local one, we refrain from detailing the nature of the space and 
assume that the solution of $(\mathrm{P})$ possesses properties sufficient for our analysis to hold. We concentrate on an analysis of $(\mathrm{P})$ for $\|u\|_{\infty} \ll 1$; however, the global behaviour is fairly well understood and described in detail in [7], [21], [34], [49]. In practical terms (P) behaves like a scalar ordinary differential equation-time-oscillatory solutions do not exist and solutions either converge to a steady state or diverge to infinity in finite or infinite time.

2.1. The linearised problem. In this subsection we consider both cases (i) and (ii). Since $f(0)=0$, the trivial solution $u(x, t) \equiv 0$ solves $(\mathrm{P})$. The linearisation of $(\mathrm{P})$ about this trivial solution is given by

$$
\begin{gathered}
v_{t}=v_{x x}+\lambda f^{\prime}(0) v, \\
v(0, t)=v(1, t)=0, \quad v(x, 0)=u_{0}(x) .
\end{gathered}
$$

This problem is readily solved by separation of variables. We seek solutions $v(x, t)=\phi(x) \psi(t)$. Then

$$
\psi(t)=\exp (\sigma t)
$$

and $\phi(x)$ satisfies

$$
\begin{gathered}
\phi_{x x}+\lambda f^{\prime}(0) \phi=\sigma \phi, \\
\phi(0)=\phi(1)=0 .
\end{gathered}
$$

The eigenvalue problem (2.1.4), (2.1.5) is fundamental to (P). First, to solve the linearised equations $(2.1 .1),(2.1 .2)$ we require the eigenvalues and eigenfunctions $\sigma$ and $\phi(x)$ of (2.1.4), (2.1.5) for fixed $\lambda$. Second, (2.1.4), (2.1.5) determines the eigenvalues $\sigma$ of the Fréchet derivative of $(\mathrm{P})$ with respect to the trivial solution. Hence, by the implicit function theorem, the eigenvalues $\lambda$ of (2.1.4), (2.1.5) for $\sigma$ satisfying $\operatorname{Re}(\sigma)=0$ define the bifurcation points from the steady solution $u(x, t) \equiv 0$ of $(\mathrm{P})$; these are the values of $\lambda$ at which nontrivial solutions to (P) branch off from $u(x, t) \equiv 0$. Thus bifurcation occurs at those values of $\lambda$ for which the growth rate of one of the modes in the linearised solution is neutral (neither decaying nor growing) in time. This is a loose definition which suffices for the simple problem $(\mathrm{P})$. The reader interested in precise conditions in the more general setting of nonlinear operator equations posed in a Banach space is referred to [8].

If $\operatorname{Im}(\sigma)=0$ the bifurcation corresponds to a branching of steady solutions, whereas $\operatorname{Im}(\sigma) \neq 0$ corresponds to the branching of periodic solutions, known as Hopf bifurcation [33]. However, (2.1.4) and (2.1.5) are in Sturm-Liouville form so that, necessarily, $\operatorname{Im}(\sigma)=0$ [28]. We prove this well-known result for the purposes of comparison with the discrete case considered in $\S 3$; this proof can be found in [12].

THEOREM 2.1.1. The eigenvalues $\sigma$ of the problem (2.1.4), (2.1.5) are real.

Proof. Multiply (2.1.4) by $\phi^{*}(x)$, the complex conjugate of $\phi(x)$, and integrate by parts. This yields

$$
\left[\sigma-\lambda f^{\prime}(0)\right] \int_{0}^{1} \phi \phi^{*} d x+\int_{0}^{1} \phi_{x} \phi_{x}^{*} d x=0 .
$$

Thus

$$
\left[\sigma-\lambda f^{\prime}(0)\right] \int_{0}^{1}|\phi|^{2} d x+\int_{0}^{1}\left|\phi_{x}\right|^{2} d x=0
$$


Taking the imaginary part gives

$$
\operatorname{Im}(\sigma) \int_{0}^{1}|\phi|^{2} d x=0
$$

since $\lambda \in R$; thus $\operatorname{Im}(\sigma)=0$ as required.

Applying the same method to the linearisation of $(\mathrm{P})$ about any steady solution shows that Hopf bifurcation cannot occur; this fact may be deduced directly from the more general result about the behaviour of $(\mathrm{P})$ described above: $(\mathrm{P})$ behaves like a scalar ordinary differential equation and cannot exhibit oscillatory behaviour.

2.2. Bifurcation analysis; steady solutions. As described in the previous subsection, the bifurcation of steady solutions of $(\mathrm{P})$ from $u(x, t) \equiv 0$ occurs at the eigenvalues $\lambda$ of the problem

$$
\phi_{x x}+\lambda f^{\prime}(0) \phi=0, \quad \phi(0)=\phi(1)=0 .
$$

Clearly, if $f^{\prime}(0)=0$ then (2.2.1) possesses no eigenfunctions; thus we consider the case $f^{\prime}(0) \neq 0$ in this and the next subsection. Without loss of generality we may scale $\lambda$ so that $f^{\prime}(0)=1$ (case $(i)$ ).

For a detailed analytic discussion of the necessary and sufficient conditions for bifurcation of steady solutions the reader is referred to [8]; for a more geometrical viewpoint see [17]. For motivation, it is sufficient to consider the following argument for the derivation of (2.2.1). We seek steady solutions $U(x)$ of $(\mathrm{P})$ with small supremum norm and set $U(x) \approx \varepsilon \phi(x)$, where $\varepsilon \ll 1$. Substituting this into $(\mathrm{P})$ and equating powers of $\varepsilon$ yields the $O(\varepsilon)$ approximation (2.2.1). Thus, for small-norm solutions of the required form to exist, we require that nontrivial solutions of (2.2.1) exist. This occurs for

$$
\lambda=(k \pi)^{2}, \quad \phi(x)=\sin (k \pi x) .
$$

Hence $U(x)$ may be approximated by $\varepsilon a_{k} \sin (k \pi x)$, for $\lambda \approx(k \pi)^{2}$. The $a_{k}$ are, as yet, undetermined.

Let us continue the expansion to the next order in $\varepsilon$, for $\lambda \approx \pi^{2}$. Since $f^{\prime \prime}(0)=0$ there are no $O\left(\varepsilon^{2}\right)$ terms in the expansion for $U(x)$. Setting

$$
U(x) \approx \varepsilon a_{1} \sin (\pi x)+\varepsilon^{3} \chi(x), \quad \lambda \approx \pi^{2}+\lambda_{0} \varepsilon^{2},
$$

we obtain

$$
\chi_{x x}+\pi^{2} \chi=-\lambda_{0} a_{1} \sin (\pi x)-\pi^{2} f^{\prime \prime \prime}(0) a_{1}^{3} \sin ^{3}(\pi x) / 6, \quad \chi(0)=\chi(1)=0 .
$$

The homogeneous version of (2.2.3) is singular (since it is precisely (2.2.1) with $\lambda=\pi^{2}$ ) so we must apply the Fredholm alternative for boundary value problems [20] to obtain the existence of a solution $\chi(x)$. This orthogonality condition yields

$$
a_{1}=0 \quad \text { or } \quad a_{1}^{2}=\frac{-6 \lambda_{0} \int_{0}^{1} \sin ^{2}(\pi x) d x}{\pi^{2} f^{\prime \prime \prime}(0) \int_{0}^{1} \sin ^{4}(\pi x) d x} .
$$

Equation (2.2.4) has two nontrivial solutions, which differ only in sign, whenever $\operatorname{sgn}\left(\lambda_{0} f^{\prime \prime \prime}(0)\right)=-1$. This is an example of a pitchfork bifurcation. This particular bifurcation structure arises because of the conditions on $f(u)$ listed in case (i). In the language of singularity theory the choice of case (i) leads to a particular normal form [17]; other classes of $f(u)$ can be considered leading to different normal forms. The case $f^{\prime \prime}(0) \neq 0$ is studied in the numerical context in [18], [52]. 
Similar pitchfork bifurcations are found for $\lambda \approx(k \pi)^{2}+\lambda_{0} \varepsilon^{2}$. The coefficients $a_{k}$ are then given by (2.2.4) with $k \pi$ replacing $\pi$ throughout:

$$
a_{k}=0 \quad \text { or } \quad a_{k}^{2}=\frac{-6 \lambda_{0} \int_{0}^{1} \sin ^{2}(k \pi x) d x}{k^{2} \pi^{2} f^{\prime \prime \prime}(0) \int_{0}^{1} \sin ^{4}(k \pi x) d x} .
$$

Piecing the information together, we obtain a local description of the steady solutions of $(\mathrm{P})$ as shown in Figs. 1 and 2 for the two cases $f^{\prime \prime \prime}(0)<0$ and $f^{\prime \prime \prime}(0)>0$. The global structure of these bifurcation diagrams is determined by the global properties of $f(u)$.

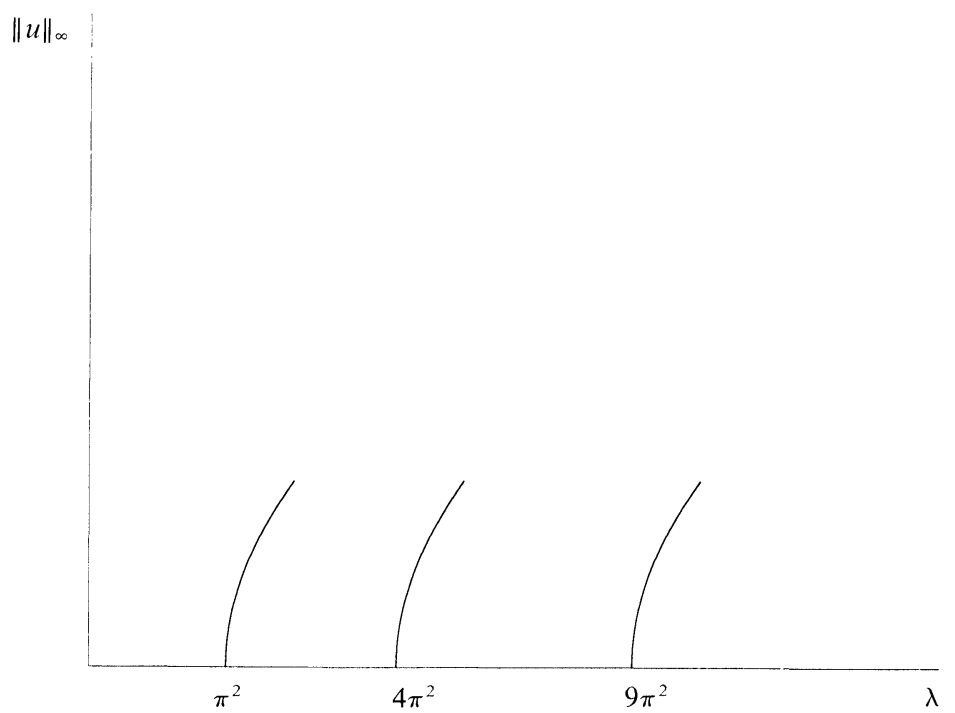

FIG. 1. Bifurcation diagram for $(\mathrm{P})$. The case $f^{\prime \prime \prime}(0)<0$.

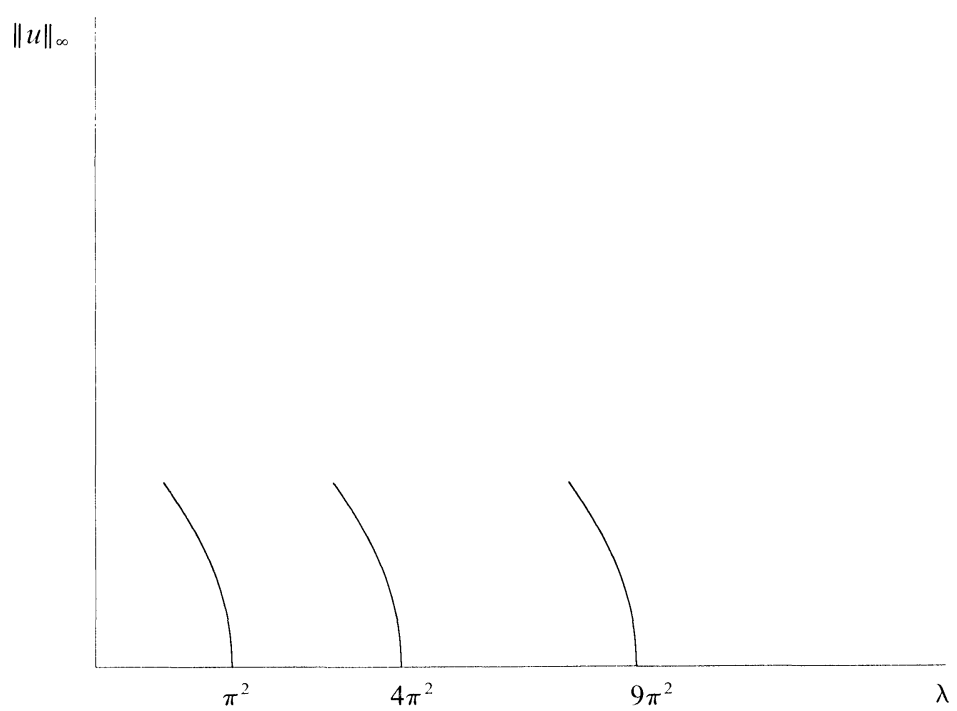

FIG. 2. Bifurcation diagram for $(\mathrm{P})$. The case $f^{\prime \prime \prime}(0)>0$. 
2.3. Weakly nonlinear analysis. In this subsection we employ the notion of the stability of steady solutions of partial differential equations. A steady solution $U(x)$ of $(\mathrm{P})$ is said to be stable in a given norm $\|\cdot\|$ if, for any $\varepsilon>0$, there exists a $\delta>0$ such that $\|u(x, 0)-U(x)\| \leqq \delta$ implies that $\|u(x, t)-U(x)\| \leqq \varepsilon$ for all $t$. In addition, we say that the solution is asymptotically stable if $\|u(x, t)-U(x)\| \rightarrow 0$ as $t \rightarrow \infty$. For problem $(\mathrm{P})$ the principle of linearised stability holds [21], [27], [49]. This means that the stability properties of a steady solution to the nonlinear problem $(\mathrm{P})$ can be inferred from the spectrum of the Frechet derivative of $(\mathrm{P})$ evaluated at the steady solution.

As in $\$ 2.2$ we assume that $f^{\prime}(0)=1$. Solving (2.1.4), (2.1.5) for $\sigma$ and $\phi(x)$, we obtain the linearised solution of $(\mathrm{P})$ :

$$
v(x, t)=\sum_{k=0}^{\infty} a_{k} \exp \left[\left(\lambda-k^{2} \pi^{2}\right) t\right] \sin (k \pi x),
$$

where the $a_{k}$ are determined by the initial conditions. For $\lambda<\pi^{2}$ we obtain $v(x, t) \rightarrow 0$ as $t \rightarrow \infty$. For $\lambda>\pi^{2}, v(x, t) \rightarrow \infty$ as $t \rightarrow \infty$. Thus $u(x, t) \equiv 0$ is a linearly stable (unstable) solution of (P) for $\lambda<\pi^{2}\left(>\pi^{2}\right)$. By the principle of linearised stability we deduce the following about (P). For $\lambda<\pi^{2}, u(x, t) \equiv 0$ is a stable solution of $(\mathrm{P})$, whereas for $\lambda>\pi^{2}$ it is not. The value $\lambda=\pi^{2}$ is the first bifurcation point from $u(x, t) \equiv 0$ (where $\operatorname{Re}(\sigma)=0$; see $\S 2.2$ ) and hence the first bifurcation point from $u(x, t) \equiv 0$ is a point of change in stability for the trivial solution.

Thus a purely linear analysis determines the stability of the solution $u(x, t) \equiv 0$ to infinitesimally small disturbances. However, to say more about the dynamics of (P) -in particular, to answer questions about finite amplitude disturbances of $u(x, t) \equiv 0$-we must take into account the form of nonlinearity $f(u)$. We do this by performing a weakly nonlinear analysis of $(\mathrm{P})$ for $\lambda \approx \pi^{2}$. This method is, in fact, a reduction of the dynamics of $(\mathrm{P})$ onto a center manifold [19]. The work in this subsection follows closely the exposition of Matkowsky [35].

The essence of weakly nonlinear analyses is to consider a modulation of the linearised solution for $\lambda \approx \pi^{2}$; the modulation is chosen on a slow time scale on which the effect of the nonlinearity in $f(u)$ plays a part. This is achieved by the method of multiple scales. Formally we set

$$
\lambda=\pi^{2}+\lambda_{0} \varepsilon^{2}
$$

and seek an expansion for $u(x, t)$ in the form

$$
u(x, t ; \varepsilon) \approx \sum_{n=1}^{\infty} v_{m}(x, t, \tau) \varepsilon^{m}
$$

where $\tau=\varepsilon^{2} t$. In keeping with the method of multiple scales we treat $t$ and $\tau$ as independent variables. The extra choice arising from the introduction of $\tau$ enables us to eliminate terms in the expansion which are secular in $t$. Abusing notation we set

$$
\frac{\partial}{\partial t} \equiv \frac{\partial}{\partial t}+\varepsilon^{2} \frac{\partial}{\partial \tau}
$$

in (P). We also assume that the initial data is of small amplitude so that

$$
u(x, 0)=\varepsilon h(x) .
$$


Substituting (2.3.2)-(2.3.4) into $(\mathrm{P})$ and equating powers of $\varepsilon$, we obtain

$$
\begin{gathered}
L v_{1} \equiv v_{1 t}-v_{1 x x}-\pi^{2} v_{1}=0, \\
L v_{2}=0, \quad L v_{3}=r_{3},
\end{gathered}
$$

where

$$
r_{3}=-v_{1 \tau}+\lambda_{0} v_{1}+\pi^{2} f^{\prime \prime \prime}(0) v_{1}^{3} / 6 .
$$

Here $v_{i}(0)=v_{i}(1)=0$. Equation (2.3.5) gives the initial conditions $v_{1}(x, 0)=h(x)$, and $v_{m}(x, 0)=0$ for $m>1$. Equation (2.3.6), together with the initial and boundary conditions, has its solution given by (2.3.1) with $\lambda=\pi^{2}$ and the $a_{k}$ functions of $\tau$ (as yet undetermined). Thus

$$
v_{1}(x, t, \tau)=a_{1}(\tau) \sin (\pi x)+\sum_{k=2}^{\infty} a_{k}(\tau) \exp \left[\left(1-k^{2}\right) \pi^{2} t\right] \sin (k \pi x),
$$

where the second (summation) term decays with $t$. The solution for $v_{2}$ is zero since $L$ is linear and the initial and boundary conditions are homogeneous.

Since the same differential operator $L$ occurs at successive orders, secular solutions $v_{3}$ may arise. We solve (2.3.7) for $v_{3}$ by Fourier decomposition, using the complete set of eigenfunctions $\sin (k \pi x)$. This gives an infinite set of coupled ordinary differential equations in $t$. To avoid secularity we remove terms constant in $t$ from the equation corresponding to $k=1$ (the mode constant in time in $v_{1}$ ). This condition can be written succinctly [35] as

$$
\lim _{T \rightarrow \infty} \frac{1}{T} \int_{0}^{T} \int_{0}^{1} \exp \left[\left(1-k^{2}\right) \pi^{2} t\right] \sin (k \pi x) r_{3} d x d t=0 \quad \text { for } k=1, \cdots
$$

The only contribution to (2.3.9) occurs with $k=1$ and comes from the first (nondecaying) term in (2.3.8) when substituted into $r_{3}$; we obtain

$$
\frac{d a_{1}}{d \tau}=\lambda_{0} a_{1}+b a_{1}^{3}
$$

where

$$
b=\frac{\pi^{2} f^{\prime \prime \prime}(0) \int_{0}^{1} \sin ^{4}(\pi x) d x}{6 \int_{0}^{1} \sin ^{2}(\pi x) d x} .
$$

The critical points of (2.3.10), $a_{1}=0$ and $a_{1}= \pm \sqrt{-\lambda_{0} / b}$, are precisely the amplitudes of the steady solutions given by (2.2.4). Equation (2.3.10) is explicitly solvable and a full description of its behaviour can be found in [12] and [35]. Combining (2.3.3) and (2.3.8) gives

$$
u(x, t) \approx \varepsilon a_{1}(\tau) \sin (\pi x)
$$

at $t \rightarrow \infty$. Using this we summarise the behaviour of $u(x, t)$ as follows:

(a) $\lambda_{0}>0, b>0$. In this case $a_{1}(\tau) \rightarrow \infty$ for finite $\tau$ for all initial data. Thus the zero solution is unstable, as predicted by linear theory (see Fig. 4 with $\lambda>\pi^{2}$ ).

(b) $\lambda_{0}<0, b>0$. In this case $a_{1}(\tau) \rightarrow 0$ as $\tau \rightarrow \infty$ for all initial data satisfying $\left|a_{1}(0)\right|<\sqrt{-\lambda_{0} / b}$. For initial values of $a_{1}(0)$ lying outside this range, $a_{1}(\tau)$ increases without bound (see Fig. $4, \lambda<\pi^{2}$ ). This is an example of a finite amplitude instability: the zero solution of $(\mathrm{P})$ is unstable to initial disturbances satisfying $\left|a_{1}(0)\right|>\sqrt{-\lambda_{0} / b}$, even though the zero solution is linearly stable. 


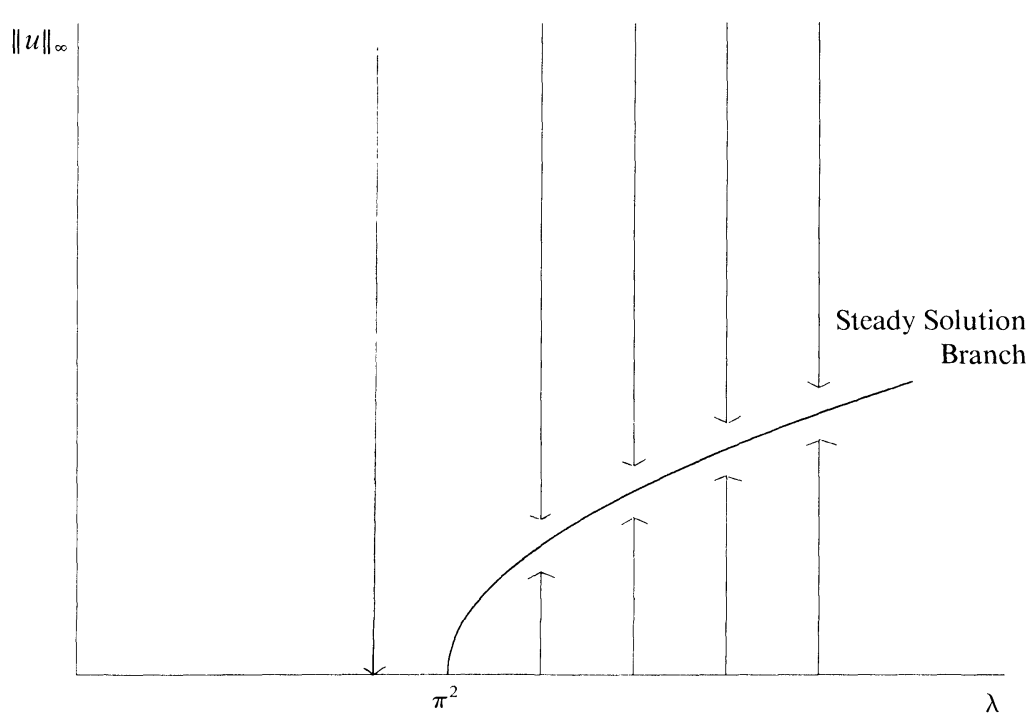

FIG. 3. Diagram showing evolution of data under (P). The case $f^{\prime \prime \prime}(0)<0$. The arrows indicate evolution in time.

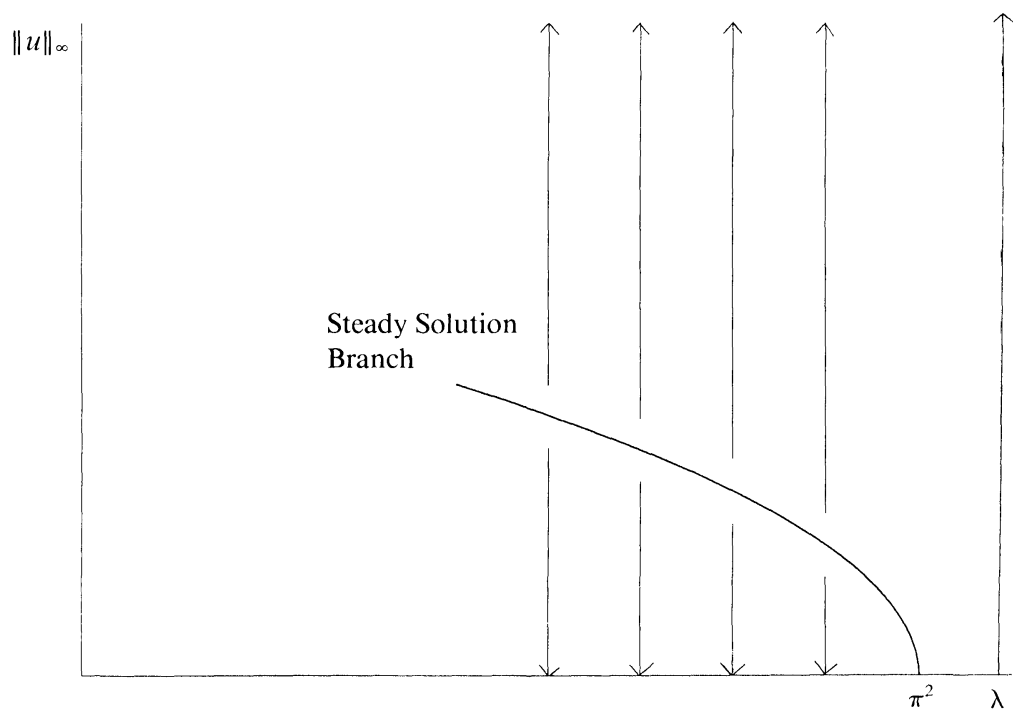

FIG. 4. Diagram showing evolution of data under (P). The case $f^{\prime \prime \prime}(0)>0$. The arrows indicate evolution in time.

(c) $\lambda_{0}>0, b<0$. In this case $a_{1}(\tau) \rightarrow \sqrt{-\lambda_{0} / b}$ for all initial data. Thus the zero solution is unstable as predicted by linear theory. Furthermore, we obtain the additional information that $u(x, t)$ evolves towards the nonzero steady state constructed in $\$ 2.2$ (see Fig. 3, $\lambda>\pi^{2}$ ).

(d) $\lambda_{0}<0, b<0$. In this case $a_{1}(\tau) \rightarrow 0$ for all initial data, confirming that the zero solution is stable for $\lambda_{0}<0$ (see Fig. $3, \lambda<\pi^{2}$ ).

2.4. Bifurcation from infinity. In this subsection we consider $f(u)$ defined by case (ii), so that $f^{\prime}(0)=0$. For simplicity, we consider (P) in the specific case $f(u)=u^{2}$. 
The discussion follows the work of Rosenblat and Davis [42], who consider a more general problem that includes the case $f(u)=u^{2}$. To standardise notation with [42] we rescale (P) by setting $\tau=\lambda t$, giving

$$
u_{\tau}=\lambda^{-1} u_{x x}+u^{2}
$$

subject to $u(0, \tau)=u(1, \tau)=0$ and some initial conditions.

Since $f^{\prime}(0)=0$ we deduce that there is no bifurcation from the trivial solution because (2.2.1) has no eigensolutions. However, (2.4.1) is characterised by a form of bifurcation from infinity. If we linearise (2.4.1) about $u(x, \tau) \equiv 0$ and solve the resulting equation by separation of variables, we obtain the linearised solution

$$
v(x, \tau)=\sum_{k=0}^{\infty} a_{k} \exp \left(-k^{2} \pi^{2} \tau / \lambda\right) \sin (k \pi x) .
$$

For $f(u)$ defined by case (i), the bifurcation points $\lambda=k^{2} \pi^{2}$ are defined by the points where the growth rates of the individual modes $\sin (k \pi x)$ composing the linearised solution (2.3.1) are neutral in time (since $\operatorname{Re}(\sigma)=0$ at such points; see $\S \S 2.1$ and 3 ). In this sense the point $\lambda=\infty$ is a candidate for a bifurcation point of infinite multiplicity in the current problem (case (ii)), since the growth rates of each mode in (2.4.2) all approach zero as $\lambda \rightarrow \infty$.

This heuristic discussion does not constitute a proof. However, in the case $f(u)=u^{2}$ it can be shown that (2.4.1) possesses a nontrivial steady solution whose supremum norm approaches zero at a rate $\lambda^{-1}$ as $\lambda \rightarrow \infty$; the details of the proof may be found in [42], together with a stability analysis of the nontrivial solution. The solution is shown to be unstable. For our purposes, it suffices to note that bifurcation from infinity is characterised by the case where the growth rates of the linearised solution all approach zero simultaneously. Furthermore, the bifurcation is subcritical and the solution is unstable; thus a finite amplitude instability occurs in this problem, even though there is no bifurcation from the trivial solution at any finite value of $\lambda$ (see [42]).

3. The discrete problem. In this section we analyse the two-level approximation (PD), known as the $\theta$-method [41], by the methods described in $\S 2$. We examine (PD) from the perspective of practical numerical stability defined in $\S 3.3$. We will show, among other things, that nonlinear numerical instability is associated with the bifurcation of solutions periodic in $n$ from steady ( $n$-independent) solutions of (PD). A clear illustration of a related phenomenon in ordinary differential equations is described in [5].

Unlike the continuous problem, the global behaviour of (PD) is not well understood. The dynamics of discretisations (which are coupled iterated maps) are generally far more complicated than the dynamics of their continuous counterparts (which are differential equations); this is certainly the case for reaction-diffusion equations. For example, if we assume that $r$ is unrestricted, the maximum principle for linear parabolic problems has no known general counterpart in the $\theta$-method for $\theta \neq 1$ (see [41])-indeed, the Crank-Nicolson method often introduces spatial oscillations not present in the differential equation. There are, however, some scattered results of a global nature about the behaviour of (PD). An important paper of Hoff [23] generalises the concept of invariant regions (see [49] and the references cited there) to discrete problems, and the method can be used to prove global results about specific discrete reaction-diffusion equations.

The work in $\$ 3.1$ is mostly well known and described in [41]; the exception is the proof of Theorem 3.1.1, which is nonstandard and presented for the purposes of 
comparison with the continuous case. The analysis in $\S 3.2$ is also well known [41], but the bifurcation theoretic presentation is new and facilitates direct comparison with the continuous case. The methods employed in $\$ 3.3$ are those of Newell [38], who generalised the method of multiple scales to difference equations. However, we consider some properties of (PD) not analysed by Newell: the evolution of arbitrary initial data and the interaction of numerical instability with the underlying dynamics of $(\mathrm{P})$.

In $\S 3.1$ we consider arbitrary functions $f(u)$. In $\$ 3.4$ we consider functions from case (ii), detailed at the start of $\S 2$. In $\$ \S 3.2$ and 3.3 we consider case (i). These assumptions on $f(u)$ simplify the analysis without changing the qualitative results. In [52] it is shown that the flip bifurcation of periodic orbits from steady solutions, characteristic of numerical instability, is generically of pitchfork type, regardless of the form of $f(u)$. However, the calculation of the coefficients in the normal forms is considerably more complicated when $f^{\prime \prime}(0) \neq 0$; the details may be found in [52].

3.1. The linearised problem. The linearisation of (PD) about $u_{j}^{n} \equiv 0$ is given by

$$
v_{j}^{n+1}-v_{j}^{n}=r \delta_{x}^{2}\left[\theta v_{j}^{n+1}+(1-\theta) v_{j}^{n}\right]+\lambda \Delta t f^{\prime}(0)\left[\theta v_{j}^{n+1}+(1-\theta) v_{j}^{n}\right],
$$

with the boundary and initial conditions

$$
v_{0}^{n}=v_{J}^{n}=0, \quad v_{j}^{0}=u_{0}(j \Delta x) .
$$

As in the continuous case, this problem may be solved by separation of variables. We seek solutions $v_{j}^{n}=\phi_{j} \psi_{n}$. Then

$$
\psi_{n}=\left[\frac{1+\sigma(1-\theta)}{1-\sigma \theta}\right]^{n},
$$

where $\sigma$ is an eigenvalue of the problem

$$
r \delta_{x}^{2} \phi_{j}+\lambda \Delta t f^{\prime}(0) \phi_{j}=\sigma \phi_{j}
$$

with

$$
\phi_{0}=\phi_{J}=0 .
$$

These equations can be written succinctly in the matrix form

$$
A \phi=\left(\lambda \Delta t f^{\prime}(0)-\sigma\right) \phi,
$$

where $A$ is a positive definite tridiagonal matrix and $\phi=\left(\phi_{1}, \phi_{2}, \cdots, \phi_{J-1}\right)^{T}$.

Equations (3.1.3)-(3.1.5) are the discrete analogues of (2.1.3)-(2.1.5) and are fundamental to $(\mathrm{PD})$. In $\S 2$ we saw that bifurcations in $(\mathrm{P})$ are determined by the eigenvalues $\lambda$ for which the growth rate $\sigma$ of a particular mode satisfies $\operatorname{Re}(\sigma)=0$, so that the linearised solution $\psi(t)$ of (2.1.3) is neutral in time $t$. Similarly we describe bifurcation in the discrete system by the condition $|\xi|=1$, where the growth rate $\xi$ is given by

$$
\xi=\frac{[1+\sigma(1-\theta)]}{[1-\sigma \theta]},
$$

so that the linearised solution (3.1.3) is neutral in $n$. In $\S 3.2$ we discuss bifurcation in (PD) further.

The following theorem is the discrete analogue of Theorem 2.1.1. This result can be proved directly by noting that (3.1.6) defines a real symmetric matrix eigenvalue problem for $\sigma$. However, we choose a discrete analogue of the proof of Theorem 2.1.1. 
THEOREM 3.1.1. The eigenvalues $\sigma$ of the problem defined by (3.1.4), (3.1.5) are real.

Proof. Multiply (3.1.4) by $\phi_{j}^{*}$, the complex conjugate of $\phi_{j}$. Summation by parts $[41$, p. 14] and boundary conditions (3.1.5) yield

$$
\left[\sigma-\lambda \Delta t f^{\prime}(0)\right] \sum_{j=1}^{J-1} \phi_{j} \phi_{j}^{*}+r \sum_{j=1}^{J-1}\left(\phi_{j+1}-\phi_{j}\right)\left(\phi_{j+1}^{*}-\phi_{j}^{*}\right)+\phi_{1} \phi_{1}^{*}=0 .
$$

Thus

$$
\left[\sigma-\lambda \Delta t f^{\prime}(0)\right] \sum_{j=1}^{J-1}\left|\phi_{j}\right|^{2}+r \sum_{j=1}^{J-1}\left|\phi_{j+1}-\phi_{j}\right|^{2}+\left|\phi_{1}\right|^{2}=0 .
$$

Taking the imaginary part and noting that $\lambda$ is real, we obtain

$$
\operatorname{Im}(\sigma) \sum_{j=1}^{J-1}\left|\phi_{j}\right|^{2}=0
$$

so that $\operatorname{Im}(\sigma)=0$ as required.

Since $\sigma$ is real we deduce from (3.1.7) that $\xi$ is real, and that bifurcation occurs for $\xi= \pm 1$ only. Equation (3.1.7) shows that if $\xi=1$ then $\sigma=0$. Hence $\xi=1$ corresponds to steady bifurcation, as in the continuous case. The case $\xi=-1$, however, has no analogy in the continuous case. In the language of dynamical systems it is known as a flip bifurcation point [19]; it corresponds to the bifurcation of solutions of (PD) with period 2 in $n$ and is purely a product of the discretisation-as we explained earlier, $(\mathrm{P})$ does not have solutions that oscillate in time. Of course we have not yet shown that $\xi=-1$ is ever achieved; in the following subsection we show that for $0 \leqq \theta<\frac{1}{2}, \xi=-1$ occurs for $J-1$ critical values of the parameter $r$.

The case $\xi=-1$ is the familiar condition for the onset of (practical) numerical instability in discretisations of linear problems [41]. In the nonlinear problem (PD), practical numerical instability is associated with the bifurcation of a branch of periodic solutions of (PD) from the trivial solution. The critical parameter value at which this bifurcation occurs is predicted by the linear theory, but, as for problem (P) in $\$ 2.3$, the behaviour of the fully nonlinear problem (PD) depends crucially on the properties of the bifurcating solution. The situation is considerably more complicated than in the continuous case, since we need to consider not only the bifurcation of discrete steady solutions (associated with $\xi=1$ ) but also the bifurcation of periodic solutions (associated with $\xi=-1$ ), which affect the numerical stability of the scheme.

3.2. Bifurcation analysis. Here $f^{\prime}(0)=1$. We solve the eigenvalue problem (3.1.4), (3.1.5) which determines the bifurcation points from $u_{j}^{n} \equiv 0$ in (PD). We seek solutions in the form $\phi_{j}=\exp (i k \pi j / J)$, for integer $k$. Substitution into (3.1.4) yields

$$
2 r(\cos (k \pi / J)-1) \exp (i k \pi j / J)=(\sigma-\lambda \Delta t) \exp (i k \pi j / J) .
$$

Thus

$$
\sigma=-4 r \sin ^{2}(k \pi / 2 J)+\lambda \Delta t .
$$

To satisfy the boundary conditions $\phi_{0}=\phi_{J}=0$ we take the imaginary part of the eigenfunction so that

$$
\phi_{j}=\sin (k \pi j / J) .
$$

For the purposes of representation on a discrete grid, we need only consider the wavenumbers $k=1, \cdots, J-1$. All other values of $k$ are merely aliases of these 
values. Thus we have constructed the complete set of eigenvectors and eigenvalues of the $(J-1)$-dimensional matrix eigenvalue problem (3.1.6).

We substitute (3.2.1) for $\sigma$ into the growth rates $\xi$ given by (3.1.7). This yields

$$
\xi_{k}=\frac{1-4 r(1-\theta) \sin ^{2}(k \pi / 2 J)+\lambda \Delta t(1-\theta)}{1+4 r \theta \sin ^{2}(k \pi / 2 J)-\lambda \Delta t \theta} \text { for } k=1, \cdots, J-1 .
$$

The condition $\xi_{k}=1$ defines the steady bifurcation points and $\xi_{k}=-1$ defines periodic bifurcation points. Let us compare the steady bifurcation points of (PD) with those of (P). Setting $\xi_{k}=1$ (note that $r=\Delta t / \Delta x^{2}$ ) gives

$$
\lambda=4 \sin ^{2}(k \pi / 2 J) / \Delta x^{2} \text { for } k=1, \cdots, J-1 .
$$

Thus the discrete model (PD) possesses only $(J-1)$ steady bifurcation points whereas the continuous model (P) has a countably infinite set (see (2.2.2)). The corresponding eigenfunctions of the two problems (compare (2.2.2) and (3.2.2)) are identical at the discrete sampling points $x=j / J=j \Delta x$. The eigenvalues, however, agree only when the wavenumber $k$ of the eigenfunction is small compared with the number of spatial meshpoints $J$. Expanding (3.2.4) for $k \ll J=\Delta x^{-1}$ gives

$$
\lambda \approx k^{2} \pi^{2}\left[1-\frac{(\Delta x k \pi)^{2}}{12}\right]
$$

Comparing this result with the continuous result (2.2.2), we deduce that for $k \ll J$ the eigenvalues are approximated to within an error of $O\left(\Delta x^{2}\right)$. But for $(J-k) / J \ll 1$, (3.2.4) gives

$$
\lambda \approx \frac{4}{\Delta x^{2}}\left[1-\frac{(J-k)^{2} \pi^{2}}{8 J^{2}}\right],
$$

which bears little resemblance to (2.2.2).

Figure 5 shows a graph comparing the loci of steady bifurcation points for $(\mathrm{P})$ and (PD) with $\Delta x=0.01$. The graph confirms the analysis above and indicates that the positions of only the first few bifurcation points in (P) are well approximated by (PD). Analogous results for spectral approximations to the differential operator $d^{2} / d x^{2}$ are discussed in [57].

It is possible to construct small-amplitude nontrivial steady solutions of (PD) in the neighbourhood of the bifurcation points (3.2.4). The analysis is the discrete analogue of the one in $\$ 2.2$. We seek expansions of the form

$$
U_{j} \approx \varepsilon a_{k} \sin (k \pi j / J)+\varepsilon^{3} \chi_{j}, \quad \lambda \approx \lambda_{k}+\lambda_{0} \varepsilon^{2} .
$$

The problem for $\chi_{j}$ arising at $O\left(\varepsilon^{3}\right)$ is singular, as in the continuous case (2.2.3). Applying the Fredholm alternative for matrices yields

$$
a_{k}=0 \quad \text { or } \quad a_{k}^{2}=\frac{-6 \lambda_{0} \sum_{j=0}^{J} \sin ^{2}(k \pi j / J) d x}{\lambda_{k} f^{\prime \prime \prime}(0) \sum_{j=0}^{J} \sin ^{4}(k \pi j / J) d x} .
$$

Here the $\lambda_{k}$ are given by (3.2.4) for $k=1, \cdots, J-1$. These values accurately approximate the amplitudes of the bifurcating trivial solutions in the continuous problem only when the $\lambda_{k}$ are good approximations of the true eigenvalues $k^{2} \pi^{2}-$ compare (2.2.5) and (3.2.5). Convergence results for the bifurcating branches are discussed in [58].

The inability of the discrete model (PD) to locate accurately all the bifurcation points of $(\mathrm{P})$, and hence the amplitudes of the bifurcating solutions, is very important 


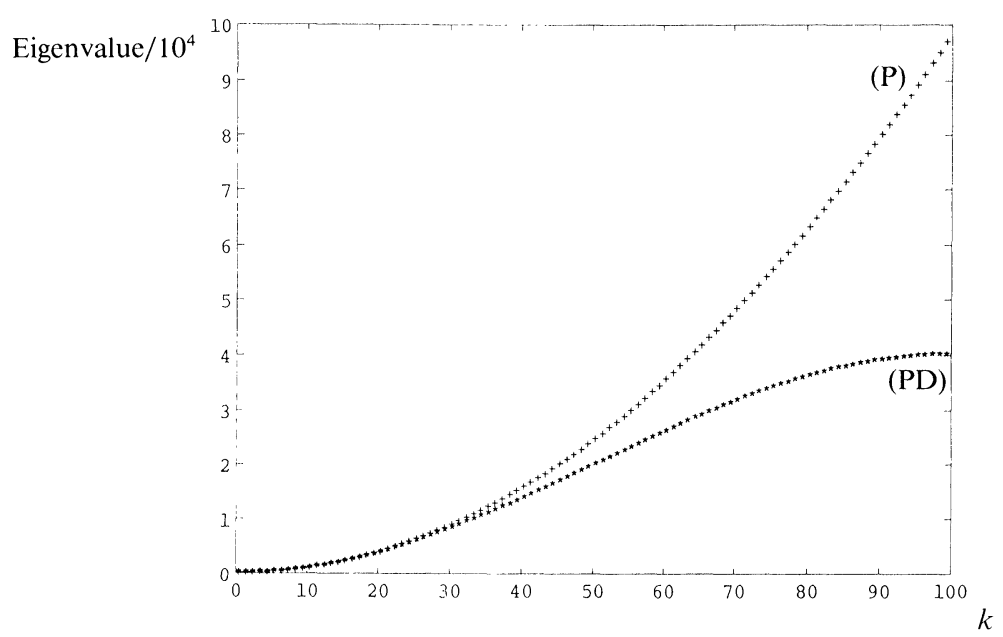

FIG. 5. Comparison of eigenvalues for (P) and (PD). $\Delta x=0.01$.

for the following reason. The dynamics of $(\mathrm{P})$ are determined by the underlying structure of the steady solution set (see [7], [21]). In any practical computation there is always a lower bound on the size of the mesh-spacing $\Delta x$, say $\Delta x_{\min }$, and a corresponding upper bound on $J\left(=\Delta x^{-1}\right)$, say $J_{\max }$. If we solve (P) approximately using (PD) then, for given $J_{\max }$, we may always choose a value of $\lambda$ above which the underlying steady solution set of (PD) is a very poor representation of that in (P), since the position of the steady bifurcation points, and hence the amplitude of the solutions, are inadequately represented. Consequently the dynamics of (P) and (PD) for sufficiently large $\lambda$ will differ substantially. We emphasise that this result is not a product of numerical instability, but merely reflects the inability of the discrete system to capture all the features of the continuous case: the steady solutions bifurcating from $\lambda=(k \pi)^{2}$ have $k-1$ zeros and thus, for given $J_{\max }$, we can always choose a $\lambda$ such that the branching steady solution cannot be resolved accurately on the grid. However, the values of $\lambda$ at which this phenomenon occurs are large and the problem may be ripe for an asymptotic analysis. For examples of such analysis applied to the steady version of (P), see Fife [15] and Norbury [39].

The converse of the phenomenon described above can also occur: a discretisation can possess steady solutions which do not correspond to any steady solution in the underlying continuous problem as the mesh is refined [4], [46]. This phenomenon is sometimes termed ghost bifurcation (see [5]). If steady solutions alone are the goal, there are two main ways of identifying these spurious solutions:

(i) To examine the bifurcation diagram for a sequence of mesh-spacings. Typically, as the mesh is refined, these spurious solutions will either move off to infinity in the bifurcation diagram or coalesce with one another at turning points.

(ii) Solutions varying on a scale comparable to the grid are frequently spurious. However, although it is relatively easy to identify spurious solutions, their presence has two important effects on the solution of (P). First, for steady solutions, it can mean that great care is needed to employ continuation procedures to prevent the nonlinear algebraic solver from converging to nonphysical solutions [47]. Second, the spurious steady solutions can affect the global dynamics of the discrete problem. (This is related to the first point, since many algebraic solvers involve a time-like iterative 
procedure.) An example of how spurious steady solutions can affect time-dependent computations is described in [51]; the numerical solution of $(\mathrm{P})$ is considered for functions $f(u)$ which lead to the development of finite-time singularities. The presence of spurious steady solutions leads to a degradation of the time-evolving problem which completely destroys the spatial structure of the solution close to the blowup time. This strictly nonlinear phenomenon occurs even for time-exact numerical methods and is caused purely by the spatial discretisation. As for the steady problem, we emphasise the importance of simulating the problem for a sequence of meshspacings to help identify phenomena that are products of the discretisation.

There is a great deal of literature concerning the numerical solution of bifurcation problems. Standard numerical schemes become singular at bifurcation points but this can be overcome by using the idea of extended systems. Numerical methods for bifurcation problems have been developed by, among others, H. Keller, A. Jepson, and A. Spence. Expository papers by these authors, and many other references, are cited in [29].

We now turn to the ghost bifurcation of periodic solutions not present in the continuous problem (P). The trivial solution $u_{j}^{n} \equiv 0$ bifurcates into periodic solutions when $\xi_{k}=-1$. From (3.2.3) this happens for

$$
r=\frac{2+\lambda \Delta t(1-2 \theta)}{4(1-2 \theta) \sin ^{2}(k \pi / 2 J)} \text { for } k=1, \cdots, J-1 .
$$

Since $r\left(=\Delta t / \Delta x^{2}\right)$ is positive, (3.2.6) determines no bifurcation points for $\frac{1}{2}<\theta \leqq 1$ (assuming that $\lambda \Delta t$ is sufficiently small that the numerator in (3.2.6) is positive), and for $\theta=\frac{1}{2}$ there are $J-1$ bifurcation points located at $r=\infty$. For $0 \leqq \theta<\frac{1}{2}$, however, there are $(J-1)$ distinct bifurcation points, the smallest of which, $r_{c}$, is given by (3.2.6) with $k=J-1$. This is the value of $r$ above which numerical instability occurs in the linearised equations (3.1.1), (3.1.2). In the following section we investigate the bearing of the value of $r_{c}$ on the fully nonlinear problem (PD). For ease of exposition we assume that $\theta=0$ although the analysis can be extended to deal with all $0 \leqq \theta<\frac{1}{2}$.

3.3. Weakly nonlinear analysis. In this subsection we employ the notion of practical numerical stability (P-stability): a solution of (PD) is said to be $\mathrm{P}$-stable if it admits no solutions that grow faster in $n \Delta t$ than solutions of $(\mathrm{P})$ do in $t$. This notion of stability is distinct from convergence stability [11], [30], [31] since it is concerned with the behaviour of (PD) for fixed values of the mesh-spacing. In particular, we shall fix $\Delta x$, and hence $J$, since the bifurcation and stability theories we employ implicitly assume a fixed-dimensional problem. With this convention the parameters $\lambda$ and $\Delta t$ are the natural distinguished or bifurcation parameters; the bifurcation parameter $r$ is defined through $\Delta t$, with $\Delta x$ fixed.

We describe the application of weakly nonlinear stability theory to (PD), in the case $\theta=0$, using the method introduced in [38]. There are two lowest critical values of the parameters $\lambda$ and $r$ at which bifurcation first occurs from the solution $u_{j}^{n} \equiv 0$ in (PD). The first is

$$
\lambda_{c}=\frac{4 \sin ^{2}(\pi / 2 J)}{\Delta x^{2}}
$$

(see (3.2.4)), the lowest value of $\lambda$ at which steady bifurcation occurs. The second is

$$
r_{c}=\frac{2}{4 \sin ^{2}((J-1) \pi / 2 J)-\lambda \Delta x^{2}},
$$


the lowest value of $r$ at which periodic bifurcation occurs. This critical value of $r$ is found by solving (3.2.6) for the minimum critical value of $\Delta t$, which occurs for $k=J-1$. Denoting this value by $\Delta t_{c}$, we define

$$
r_{c}=\frac{\Delta t_{c}}{\Delta x^{2}} \text {. }
$$

We emphasise that we expect this value of $r$ to be associated with numerical instability in (PD), since it corresponds to $\xi_{J-1}=-1$.

There are three possible parameter ranges in which we can perform weakly nonlinear analyses in (PD):

(a) $r \approx r_{c}, \lambda<\lambda_{c}$, and $\left(\lambda-\lambda_{c}\right)=O(1)$

(b) $\lambda \approx \lambda_{c}, r<r_{c}$, and $\left(r-r_{c}\right)=O(1)$;

(c) $\lambda \approx \lambda_{c}$ and $r \approx r_{c}$.

Case (a) is essentially that considered by Newell [38], although he did not consider arbitrary initial disturbances, but only initial data proportional to the most unstable mode $(\sin ((J-1) \pi j / J)$ in this case). Following Matkowsky's work [35] on the continuous problem (see $\$ 2.3$ ), we treat arbitrary initial disturbances. Case (b) is the discrete analogue of the analysis in $\$ 2.3$; as the analysis is similar, we omit the details and present only the results. Case (c), describing the interaction of numerical instability with the dynamics of the underlying partial differential equation, has not been considered before, and we analyse it in some detail.

Case (a). We seek the solution of (PD) for $r$ close to the critical value defined by (3.3.2). Since we are considering a problem of fixed dimension (that is, $\Delta x$ is fixed), any perturbation of $r$ necessarily induces a perturbation of $\Delta t$. Hence we set

$$
r=r_{c}+r_{0} \varepsilon^{2}, \quad \Delta t=\Delta t_{c}+r_{0} \Delta x^{2} \varepsilon^{2} .
$$

We assume that $\left(\lambda-\lambda_{c}\right)=O(1)$ and $\lambda<\lambda_{c}$. Following the analysis of Matkowsky outlined in $\$ 2.3$, we seek an expansion for $u_{j}^{n}$ in the form

$$
u_{j}^{n} \approx \sum_{m=1}^{\infty} v_{m}(n, j, \eta) \varepsilon^{m},
$$

where $\eta$ is a slowly varying $n$-variable, treated as independent. The extra freedom obtained by introducing $\eta$ enables us to suppress secularity in $n$. We choose small amplitude initial data of the form $u_{j}^{0}=\varepsilon h(j \Delta x)$.

We assume that $v_{1}(n, j, \eta)$ has the form of the linearised solution with coefficients varying weakly in $n$, so that

$$
v_{1}(n, j, \eta)=\sum_{k=1}^{J-1} \xi_{k}^{n} \sin (k \pi j / J) A_{k}(n ; \varepsilon),
$$

where $A_{k}(n ; \varepsilon) \equiv a_{k}(\eta)$. The $\xi_{k}$ are defined by (3.2.3) with $\theta=0, r=r_{c}$, and $\Delta t=\Delta t_{c}$ so that

$$
\xi_{k}=1-4 r_{c} \sin ^{2}(k \pi / 2 J)+\lambda \Delta t_{c} \quad \text { for } k=1, \cdots, J-1 .
$$

Thus $\xi_{J-1}=-1$ and $\left|\xi_{k}\right|<1$ for $k=1, \cdots, J-2$. We now make the assumption of multiple scales, namely that

$$
A_{k}(n+1 ; \varepsilon)-A_{k}(n ; \varepsilon)=\varepsilon^{2}\left[a_{k}(\eta+1)-a_{k}(\eta)\right] .
$$

This is essentially assumption (2.12) of [38]. The precise meaning of the variable $\eta$ is not as clear as that of its continuous counterpart $\tau$ in $\S 2.3$, since (PD) is only 
defined on a discrete grid (representing discrete time intervals) and fractional multiples of the timestep have no meaning; this matter is addressed further in [45]. For our purposes it is sufficient to consider (3.3.6) as the defining relationship for $a_{k}(\eta+1)$ and to note that the $a_{k}$ 's can be eliminated in favour of the $A_{k}$ 's in the final recurrence relation (3.3.10). Identical results are obtained by using the discrete multiple-scales operators defined in [45].

Substituting (3.3.3)-(3.3.5) into (PD) and equating successive powers of $\varepsilon$, we obtain

$$
\begin{gathered}
L_{d} v_{1}=L_{d} v_{2}=0, \\
L_{d} v_{3}=\sum_{k=1}^{J-1}\left[\xi_{k}^{n+1}\left(a_{k}(\eta)-a_{k}(\eta+1)\right) \sin (k \pi j / J)+\xi_{k}^{n} r_{0} \delta_{x}^{2} \sin (k \pi j / J) a_{k}(\eta)\right] \\
+\lambda r_{0} \Delta x^{2}\left[\sum_{k=1}^{J-1} \xi_{k}^{n} \sin (k \pi j / J) a_{k}(\eta)\right] \\
+\lambda \Delta t_{c^{\prime}} f^{\prime \prime \prime}(0)\left[\sum_{k=1}^{J-1} \xi_{k}^{n} \sin (k \pi j / J) a_{k}(\eta)\right]^{3} / 6,
\end{gathered}
$$

with $\left(v_{m}\right)_{0}=\left(v_{m}\right)_{J}=0$. Here

$$
L_{d} v_{j}^{n}=v_{j}^{n+1}-v_{j}^{n}-r_{c} \delta_{x}^{2} v_{j}^{n}-\lambda \Delta t_{c} v_{j}^{n} .
$$

The initial conditions are $\left(v_{1}\right)_{j}^{0}=h(j \Delta x)$ and $\left(v_{2}\right)_{j}^{0}=\left(v_{3}\right)_{j}^{0}=0$. Thus $v_{2}$ is identically zero.

As in the continuous case (see \$2.3), secularity may occur since the finite difference operator $L_{d}$ occurs at successive orders. We solve (3.3.8) by Fourier decomposition of $v_{3}$ in terms of the eigenfunctions $\sin (k \pi j / J)$. This gives a set of $J-1$ coupled recurrence relations in $n$. To suppress secularity we remove terms proportional to $(-1)^{n}$ from the recurrence relation corresponding to $k=J-1$ (the mode proportional to $(-1)^{n}$ in $\left.v_{1}\right)$. This requires the discrete analogue of (2.3.9), namely

$$
\lim _{N \rightarrow \infty} \frac{1}{N} \sum_{n=1}^{N} \sum_{j=0}^{J} \xi_{k}^{n} \sin (k \pi j / J) r_{3}=0 \quad \text { for } k=1, \cdots, J-1 .
$$

Here $r_{3}$ denotes the right-hand side of (3.3.8).

Since $\left|\xi_{k}\right|<1$ for $k \neq J-1$, this condition yields nothing except from the mode corresponding to $k=J-1$. Note that

$$
\delta_{x}^{2} \sin (k \pi j / J)=-4 \sin ^{2}(k \pi / 2 J) \sin (k \pi j / J) ;
$$

condition (3.3.9) gives us

$$
a_{J-1}(\eta+1)-a_{J-1}(\eta)=\left[4 r_{0} \sin ^{2}((J-1) \pi / 2 J)-\lambda r_{0} \Delta x^{2}\right] a_{J-1}(\eta)-b a_{J-1}^{3}(\eta),
$$

where

$$
b=\frac{\lambda \Delta t_{c} f^{\prime \prime \prime}(0) \sum_{j=0}^{J} \sin ^{4}((J-1) \pi j / J)}{6 \sum_{j=0}^{J} \sin ^{2}((J-1) \pi j / J)} .
$$

By (3.3.2) this may be written as

$$
a_{J-1}(\eta+1)-a_{J-1}(\eta)=\frac{2 r_{0}}{r_{c}} a_{J-1}(\eta)-b a_{J-1}^{3}(\eta) .
$$

This may be rewritten in terms of $A_{J-1}(n+1 ; \varepsilon)$ by use of (3.3.6). 
Equation (3.3.10) describes the (slow) evolution of the amplitude $a_{J-1}(\eta)$. The modes associated with all other amplitudes are decaying $\left(\left|\xi_{k}\right|<1\right)$; therefore (3.3.10) determines the asymptotic behaviour of (PD) for $\left|u_{j}^{n}\right| \ll 1$ and $r \approx r_{c}$. A thorough discussion of the behaviour of (3.3.10) is in [38] and the results are summarised below (for $a(\eta) \equiv a_{J-1}(\eta)$ ), after which we describe some illustrative numerical results.

$r_{0}<0$. In this case $r$ is below the linearised numerical instability threshold $r=r_{c}$. For $b>0$ all solutions of (3.3.10) tend to zero as $\eta \rightarrow \infty$. Hence the nonlinear problem acts in a stable fashion, as predicted by linear theory. If $b<0$ then the evolution of $a(\eta)$ depends critically upon $a(0)$ : if $|a(0)|<\gamma$ then $a(\eta) \rightarrow 0$ as $\eta \rightarrow \infty$. Otherwise, $|a(\eta)| \rightarrow \infty$ as $\eta \rightarrow \infty$. Here $\gamma$ is the nonzero critical point of (3.3.10), namely

$$
\gamma^{2}=\frac{2 r_{0}}{b r_{c}}
$$

Thus finite amplitude numerical instability is possible in the nonlinear problem for values of $r$ beneath the critical linearised prediction of $r_{c}$, provided that the initial excitation of the unstable mode is sufficiently large.

$r_{0}>0$. In this case $r$ is above the linearised numerical stability threshold. For $b<0$ all solutions satisfy $|a(\eta)| \rightarrow \infty$ as $\eta \rightarrow \infty$, which is the natural manifestation of numerical instability in parabolic problems, namely the unbounded growth of a highly oscillatory spatial mode; note, however, that the instability is far more explosive than the linear theory $(b=0)$ predicts. If $b>0$, then the critical point $\gamma$ can be an attractor and some solutions of (3.3.10) evolve so that $|a(\eta)| \rightarrow \gamma$ as $\eta \rightarrow \infty$. In this case we deduce from (3.3.4)-(3.3.6) that

$$
u_{j}^{n} \approx \varepsilon(-1)^{n} \sin [(J-1) \pi j / J] \gamma
$$

as $\eta$ (and hence $n) \rightarrow \infty$. Thus $u_{j}^{n}$ evolves towards a solution oscillatory in $n$. This stable oscillatory state is purely a product of the discretisation. It is not the standard manifestation of a numerical instability since its growth is bounded-here the numerical instability is inhibited by the nonlinearity and the two effects balance to produce an oscillation.

In summary of case (a), we note that practical numerical instability in (PD) is associated with the bifurcation of a periodic orbit from the trivial solution $u_{j}^{n} \equiv 0$. The manifestation of the numerical instability depends crucially on the properties of this periodic orbit. If the periodic orbit repels data starting in its vicinity, then the numerical instability occurs in the familiar fashion as the unbounded growth of an oscillatory spatial mode; in addition, finite amplitude numerical instability occurs at values of $r$ beneath those predicted by the linear theory. This is demonstrated in Fig. 6, which shows the solution of (PD) with $f(u)=u-u^{3}$. The parameters are chosen so that $\Delta x=0.01, \lambda=1.0$, and $r<r_{c}$. Thus the numerical method is operating below the linear stability limit. The initial data is proportional to the most unstable mode $\sin ((J-1) \pi j / J)$. The initial evolution of the mode is governed by (3.3.10). Eventually other modes are stimulated and a nonlinear focusing occurs. Soon after Fig. $6 \mathrm{~d}$ the scheme blows up. It may be shown that the $L_{2}$-norm of the true solution of $(\mathrm{P})$ is a monotonic decreasing function satisfying, for $t>0$,

$$
\|u(x, t)\|<\|u(x, 0)\| e^{\left(1-\pi^{2} / 4\right) t} .
$$

Figure 7 was generated from the same parameter set as Fig. 6 but with a parabolic profile as initial data. While the asymptotic behaviour is correct, the transient behaviour is entirely spurious: the solution forms four interior zeros in one timestep. The spatial structure shown in Fig. $7 \mathrm{c}, \mathrm{d}$ is characteristic of subcritical numerical instability and it can be explained by a modified equations analysis (see [52]). 


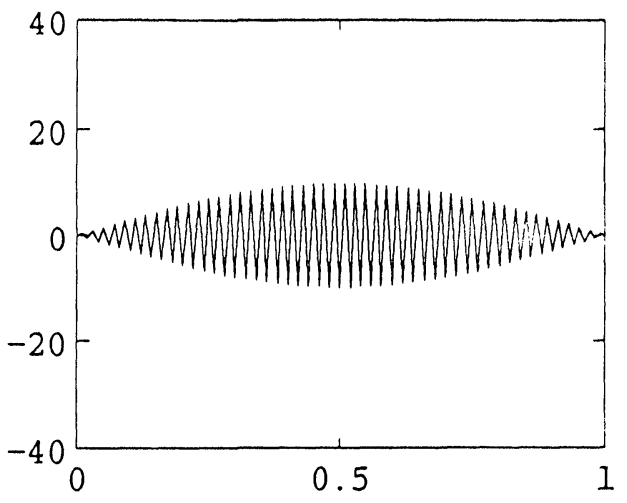

(a)

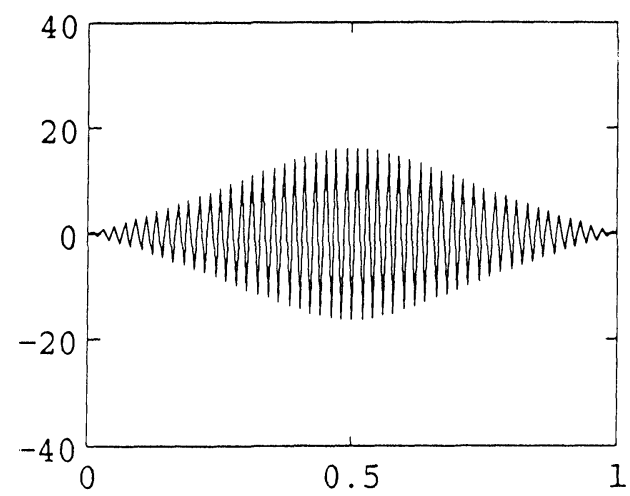

(c)

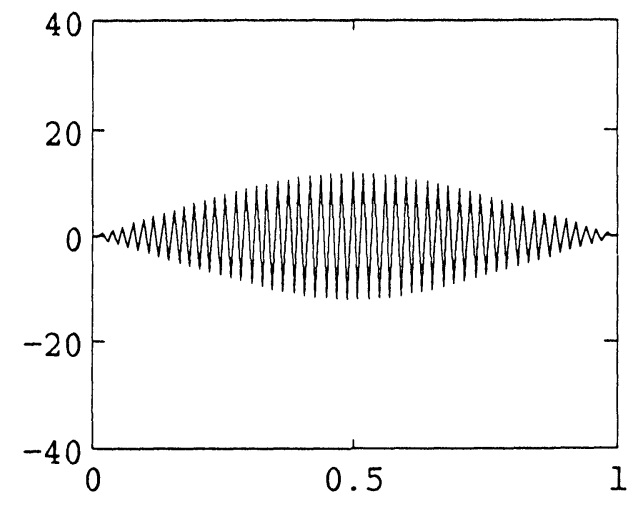

(b)

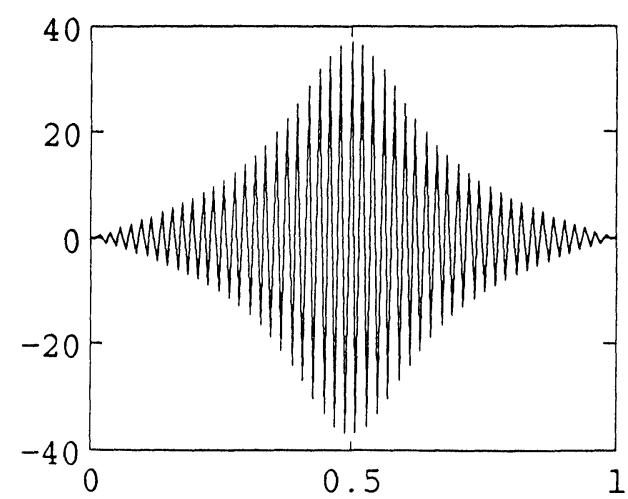

(d)

FIG. 6. Solution of (PD) for $r<r_{c}$. (a) $t=0$. (b) $t=70 \Delta t$. (c) $t=140 \Delta t$. (d) $t=210 \Delta t$.

If the periodic orbit is an attractor for data starting in its vicinity, however, then the numerical instability of the scheme is far more subtle since its manifestation is bounded periodic behaviour (in $n$ ). This is demonstrated in Fig. 8, which shows the solution of (PD) with $f(u)=u+u^{3}, \Delta x=0.05, \lambda=1.0$, and $r>r_{c}$. For large $n$ the solution oscillates between the profile shown in Fig. 8d and its negative at successive timesteps. Figure $8 \mathrm{e}$ shows the $l_{2}$-norm of the solution plotted against time; initially the amplitude of the solution decays, until the linear instability magnifies the unstable high wavenumber component significantly. Thereafter, the amplitude continues to grow until nonlinearity balances the linear instability and an equilibrium is reached.

Case (b). This case concerns the solution of (PD) for $\lambda=\lambda_{c}+\lambda_{0} \varepsilon^{2}$, on the assumption that $\left(r-r_{c}\right)=O(1)$ and $r<r_{c}$. The analysis is analogous to that considered in case (a) and we omit the details. We seek an expansion for $u_{j}^{n}$ in form (3.3.4), where $v_{1}(n, j, \eta)$ is given by (3.3.6). The $\xi_{k}$ are defined by (3.2.3) with $\theta=0$ and $\lambda=\lambda_{c}$ (see (3.3.1)), so that

$$
\xi_{k}=1-4 r \sin ^{2}(k \pi / 2 J)+\lambda_{c} \Delta t
$$

Thus $\xi_{1}=1$, and $\left|\xi_{k}\right|<1$, for $k=2, \cdots, J-1$.

A similar analysis to that in case (a) shows that

$$
v_{1}(n, j, \eta)=\sum_{k=1}^{J-1} \xi_{k}^{n} \sin (k \pi j / J) a_{k}(\eta),
$$




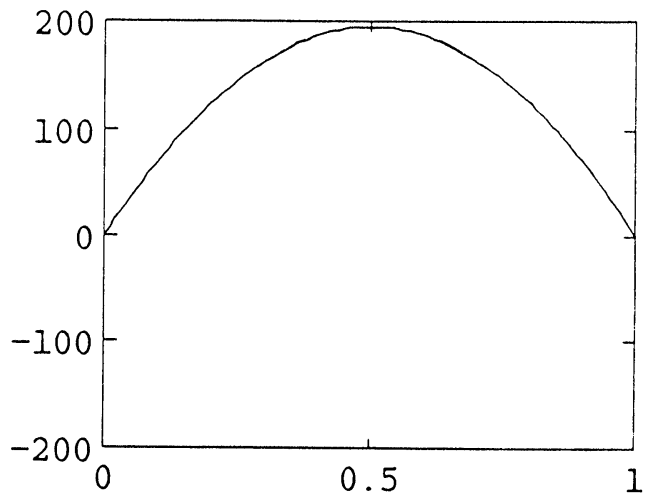

(a)

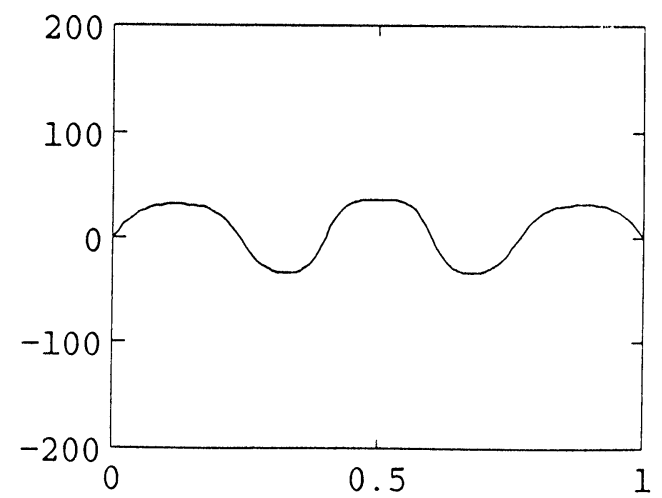

(c)

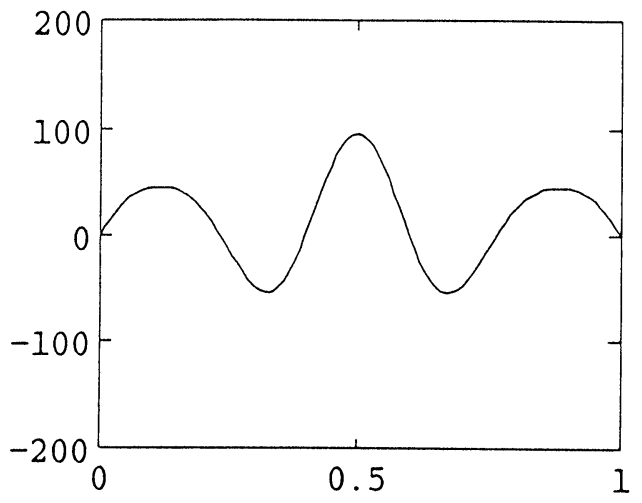

(b)

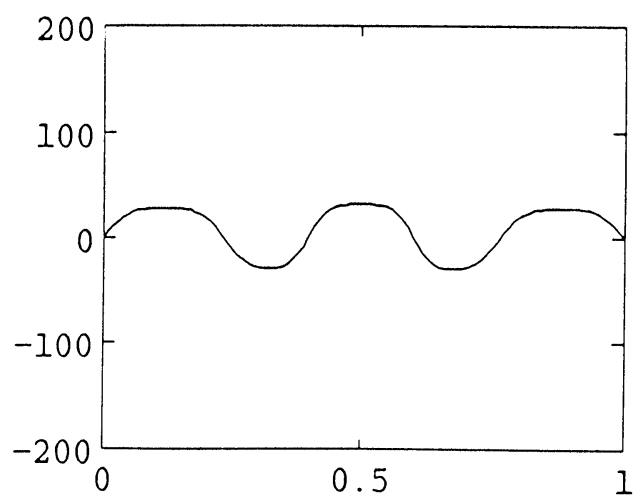

(d)

FIG. 7. Solution of (PD) for $r<r_{c}$. (a) $t=0$. (b) $t=\Delta t$. (c) $t=2 \Delta t$. (d) $t=3 \Delta t$.

where $a_{1}(\eta)$, the amplitude of the nondecaying mode, is governed by

$$
a_{1}(\eta+1)-a_{1}(\eta)=\Delta t \lambda_{0} a_{k}(\eta)+b a_{k}^{3}(\eta),
$$

with

$$
b=\frac{\lambda_{c} \Delta t f^{\prime \prime \prime}(0) \sum_{j=1}^{J-1} \sin ^{4}(\pi j / J)}{6 \sum_{j=1}^{J-1} \sin ^{2}(\pi j / J)} .
$$

Thus (3.3.12) is the discrete analogue of (2.3.10) - indeed, if we rewrite (3.3.12) in terms of $A_{1}(n ; \varepsilon)$ by using (3.3.6) and take the limits $\Delta x \rightarrow 0$ and $\Delta t \rightarrow 0,(2.3 .10)$ is recovered-and the behaviour is essentially as in the continuous case, summarised in Figs. 3 and 4. Thus, in the neighborhood of the steady bifurcation point $\lambda_{c}=\pi^{2}$ in $(\mathrm{P})$, the behaviour of $(\mathrm{PD})$ and $(\mathrm{P})$ is similar, since $\lambda \approx \pi^{2}$. This is true for the other steady bifurcation points except that, as we noted earlier, the approximation of the position of the bifurcation points $\lambda=(k \pi)^{2}$ and the corresponding amplitudes of the bifurcating solutions deteriorate rapidly with increasing $k$ (for fixed $J$ ).

Case (c). We now analyse the discrete problem (PD) in the case where both $\lambda$ and $r$ are close to their critical values $\lambda_{c}$ and $r_{c}$. The method is an extension of Newell's presented in case (a). However, the eigenvalue of the linearised operator (3.1.4), (3.1.5) determining bifurcation is of multiplicity 2 ; the analysis is complicated by this fact. An analysis of bifurcation from a double eigenvalue in the partial differential equation 


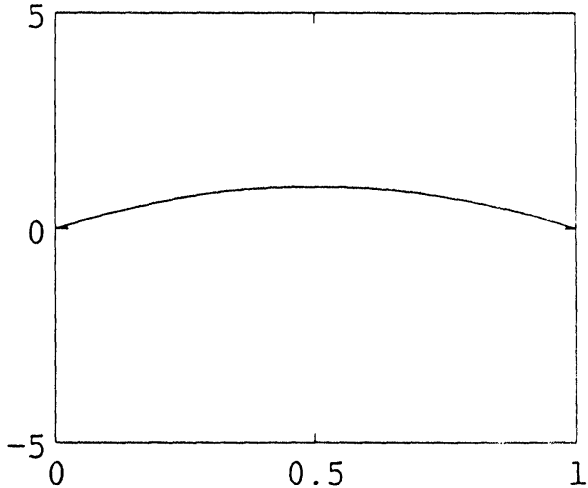

(a)

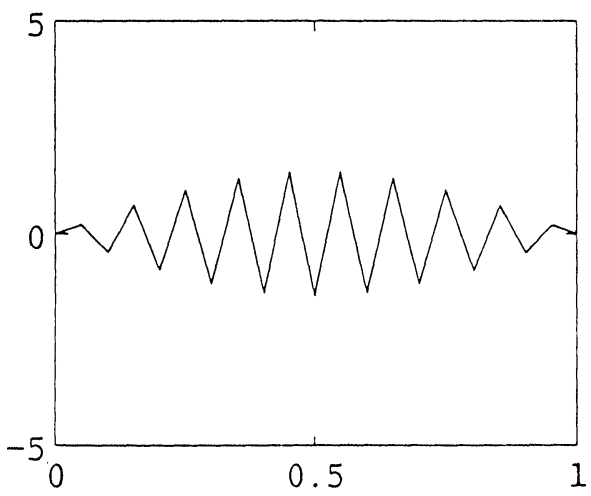

(c)

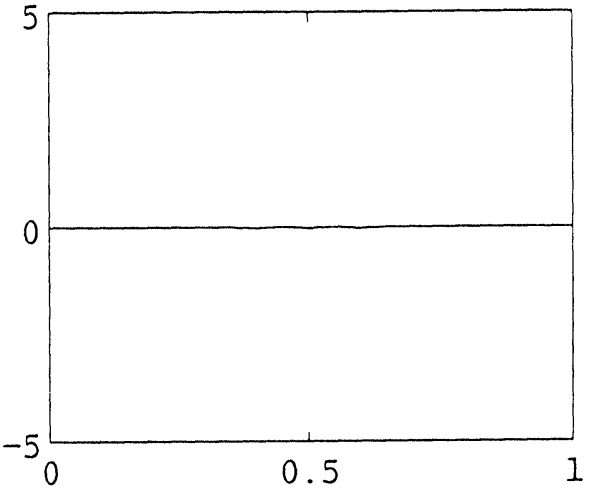

(b)

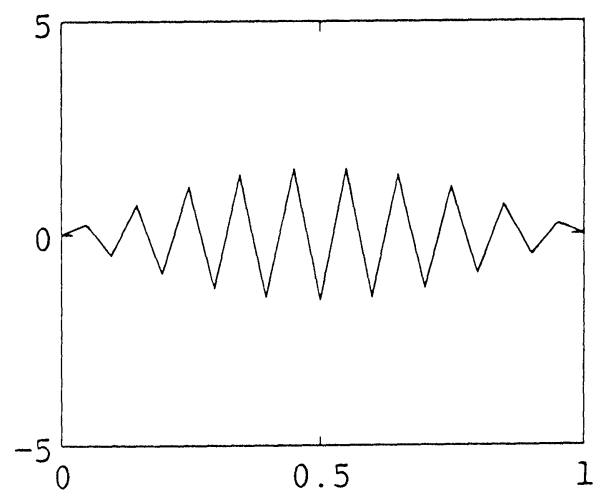

(d)

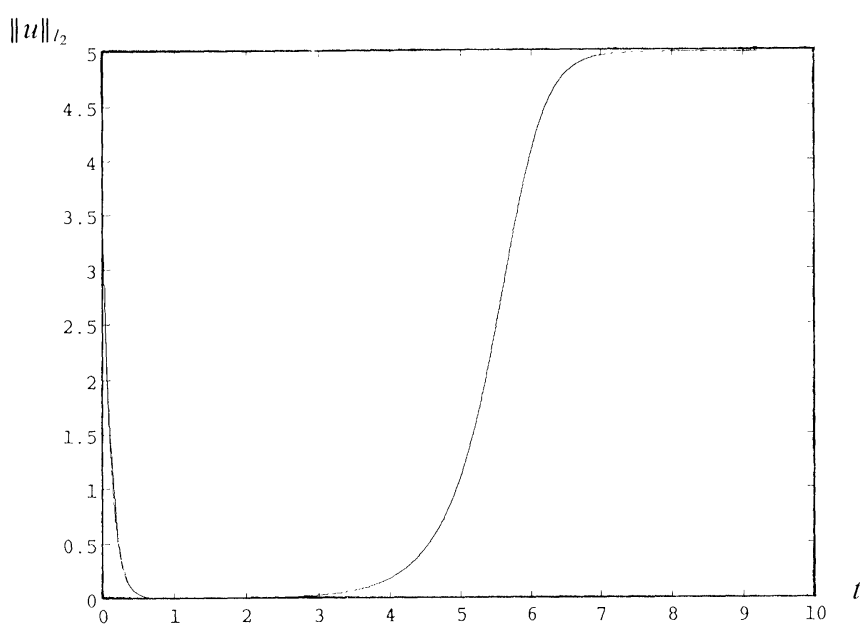

(e)

Fig. 8. Solution of (PD) for $r>r_{c}$. (a) $t=0$. (b) $t=2500 \Delta t$. (c) $t=5000 \Delta t$. (d) $t=7500 \Delta t$. (e) Convergence to a spurious equilibrium; $\|u(x)\|_{l_{2}}$ versus $t$ for $r>r_{c}$.

case can be found in Keener [26]. The existence of a double eigenvalue is usually associated with secondary bifurcation [1], and we show that this is indeed the case here. We show that the onset of numerical instability in the nonlinear problem (PD) is associated not only with bifurcation of periodic orbits from the trivial solution (as 
in case (a)), but also with bifurcation of periodic orbits from nontrivial steady state branches.

We seek an expansion for the solution $u_{j}^{n}$ of (PD) under assumptions (3.3.4)(3.3.6). In addition we now assume that

$$
\lambda=\lambda_{c}+\lambda_{0} \varepsilon^{2}, \quad \Delta t=\Delta t_{c}+r_{0} \Delta x^{2} \varepsilon^{2}, \quad r=r_{c}+r_{0} \varepsilon^{2},
$$

so that the growth rates $\xi_{k}$ (from (3.2.3) with $\theta=0$ ) satisfy $\xi_{1}=1, \xi_{J-1}=-1$, and $\left|\xi_{k}\right|<1$ for $k=2, \cdots, J-2$. Here $r_{c}$ is given by (3.3.2) with $\lambda=\lambda_{c}$. Substituting (3.3.4)-(3.3.6) and (3.3.13) into (PD) and equating successive powers of $\varepsilon$ yield

$$
\begin{aligned}
& L_{d} v_{1}=L_{d} v_{2}=0, \\
& \begin{aligned}
L_{d} v_{3}= & \sum_{k=1}^{J-1}\left[\xi_{k}^{n+1}\left(a_{k}(\eta)-a_{k}(\eta+1)\right) \sin (k \pi j / J)\right. \\
& \left.\quad+\xi_{k}^{\prime \prime}\left(\lambda_{c} r_{0} \Delta x^{2}+\lambda_{0} \Delta t_{c}-4 r_{0} \sin ^{2}(k \pi / 2 J)\right) a_{k}(\eta) \sin (k \pi j / J)\right] \\
& +\lambda_{c} \Delta t_{c} f^{\prime \prime \prime}(0)\left[\sum_{k=1}^{J-1} \xi_{k}^{\prime \prime} a_{k}(\eta) \sin (k \pi j / J)\right]^{3} / 6,
\end{aligned}
\end{aligned}
$$

where $L_{d}$ is identical to the definition beneath (3.3.8), except that $\lambda_{c}$ replaces $\lambda$. Denoting the right-hand side of (3.3.14) by $r_{3}$ we may write

$$
\begin{aligned}
r_{3}= & {\left[a_{1}(\eta)-a_{1}(\eta+1)\right] \sin (\pi j / J)+(-1)^{n+1}\left[a_{J-1}(\eta)-a_{J-1}(\eta+1)\right] \sin \left((J-1) \frac{\pi j}{J}\right) } \\
& +\left[\lambda_{c} r_{0} \Delta x^{2}+\lambda_{0} \Delta t_{c}-4 r_{0} \sin ^{2}(\pi / 2 J)\right] a_{1}(\eta) \sin (\pi j / J) \\
& +\left[\lambda_{c} r_{0} \Delta x^{2}+\lambda_{0} \Delta t_{c}-4 r_{0} \sin ^{2}((J-1) \pi / 2 J)\right](-1)^{n} a_{J-1}(\eta) \sin ((J-1) \pi j / J) \\
& +\lambda_{c} \Delta t_{c} f^{\prime \prime \prime}(0)\left[a_{1}^{3}(\eta) \sin ^{3}(\pi j / J)+(-1)^{3 n} a_{J-1}^{3}(\eta) \sin ^{3}((J-1) \pi j / J)\right. \\
& +(-1)^{\prime \prime} 3 a_{1}^{2}(\eta) a_{J-1}(\eta) \sin ^{2}(\pi j / J) \sin ((J-1) \pi j / J) \\
& \left.+3 a_{1}(\eta) a_{J-1}^{2}(\eta) \sin (\pi j / J) \sin ^{2}((J-1) \pi j / J)\right] / 6+r^{*},
\end{aligned}
$$

where

$$
\lim _{N \rightarrow \infty} \frac{1}{N} \sum_{n=1}^{N} \sum_{j=0}^{J} r^{*}=0 .
$$

Thus we have separated the terms that cause secular growth from those that do not (contained in $r^{*}$ ).

As in case (a), we solve (3.3.14) by Fourier decomposition, choosing the $a_{k}(\eta)$ to suppress the occurrence of terms secular in $n$. Thus, in the decomposition of $v_{3}$ corresponding to $k=1$ we remove terms constant in $n$ (since the mode with $k=1$ is constant in $n$ in $v_{1}$ ), and in the decomposition corresponding to $k=J-1$ we remove terms proportional to $(-1)^{n}$ (since the mode with $k=J-1$ is proportional to $(-1)^{n}$ in $\left.v_{1}\right)$. This yields the following pair of coupled recurrence relations for $a_{1}(\eta)$ and $a_{J-1}(\eta)$ :

$$
\begin{gathered}
a_{1}(\eta+1)-a_{1}(\eta)=d_{1} a_{1}(\eta)+b_{1} a_{1}^{3}(\eta)+b_{2} a_{1}(\eta) a_{J-1}^{2}(\eta), \\
a_{J-1}(\eta+1)-a_{J-1}(\eta)=d_{2} a_{J-1}(\eta)+b_{3} a_{J-1}^{3}(\eta)+b_{4} a_{J-1}(\eta) a_{1}^{2}(\eta) .
\end{gathered}
$$


The coefficients are given by

$$
\begin{aligned}
& d_{1}=\lambda_{c} r_{0} \Delta x^{2}+\lambda_{0} \Delta t_{c}-4 r_{0} \sin ^{2}(\pi / 2 J), \\
& d_{2}=4 r_{0} \sin ^{2}((J-1) \pi / 2 J)-\lambda_{0} \Delta t_{c}-\lambda_{c} r_{0} \Delta x^{2}=\frac{2 r_{0}}{r_{c}}-\lambda_{0} \Delta t_{c}, \\
& b_{1}=\lambda_{c^{\prime}} \Delta t_{c} f^{\prime \prime \prime}(0) \frac{\sum_{j=1}^{J-1} \sin ^{4}(\pi j / J)}{6 \sum_{j=1}^{J-1} \sin ^{2}(\pi j / J)}, \\
& b_{2}=\lambda_{c} \Delta t_{c} f^{\prime \prime \prime}(0) \frac{\sum_{j=1}^{J-1} \sin ^{2}(\pi j / J) \sin ^{2}((J-1) \pi j / J)}{2 \sum_{j=1}^{J-1} \sin ^{2}(\pi j / J)}, \\
& b_{3}=-\lambda_{c} \Delta t_{c} f^{\prime \prime \prime}(0) \frac{\sum_{j=1}^{J-1} \sin ^{4}((J-1) \pi j / J)}{6 \sum_{j=1}^{J-1} \sin ^{2}((J-1) \pi j / J)}, \\
& b_{4}=-\lambda_{c} \Delta t_{c} f^{\prime \prime \prime}(0) \frac{\sum_{j=1}^{J-1} \sin ^{2}(\pi j / J) \sin ^{2}((J-1) \pi j / J)}{2 \sum_{j=1}^{J-1} \sin ^{2}((J-1) \pi j / J)} .
\end{aligned}
$$

Since $\left|\xi_{k}\right|<1$ for $k=2, \cdots, J-2, \xi_{1}=1$, and $\xi_{J-1}=-1$ we deduce from (3.3.4), (3.3.5) that for large $n$ (PD) has the approximate solution

$$
u_{j}^{n} \approx \varepsilon v_{1}(n, j, \eta) \approx \varepsilon a_{1}(\eta) \sin (\pi j / J)+\varepsilon(-1)^{n} a_{J-1}(\eta) \sin ((J-1) \pi j / J) .
$$

Thus the behaviour of $a_{1}(\eta)$ and $a_{J-1}(\eta)$ as described by (3.3.15), (3.3.16) governs the evolution of $u_{j}^{\prime \prime}$. A complete analysis of (3.3.15), (3.3.16) is not possible in a short space and we confine ourselves to a brief discussion.

Equations (3.3.15) and (3.3.16) possess four critical points (solutions independent of $\eta$ ), namely

$$
(0,0)\left(0, \sqrt{\frac{-d_{2}}{b_{3}}}\right)\left(\sqrt{\frac{-d_{1}}{b_{1}}, 0}\right)\left(\sqrt{\frac{d_{2} b_{2}-d_{1} b_{3}}{b_{1} b_{3}-b_{2} b_{4}}}, \sqrt{\frac{d_{1} b_{4}-d_{2} b_{4}}{b_{1} b_{3}-b_{2} b_{4}}}\right) .
$$

The second and third critical points occur as a bifurcation from the first (trivial) critical point; the fourth occurs as a bifurcation from either the second or the third critical point. In terms of the solution of (PD), the second critical point represents a mode that oscillates in $n$, the third a stationary solution, and the fourth a mixture of the two. The existence of the mixed mode is important, since it demonstrates that the onset of numerical instability in (PD) is associated with the bifurcation of periodic orbits (in $n$ ) from a nontrivial solution. Of course our analysis is restricted to the neighbourhood of $\lambda \approx \lambda_{c}$ and $r \approx r_{c}$; but, by continuation to other parameter values, we deduce that the secondary bifurcation of numerically destabilising periodic orbits from steady solutions is a generic occurrence in (PD).

The number of possible bifurcation diagrams for (3.3.15), (3.3.16) is large due to the number of parameters. We depict two, showing the secondary bifurcation of the mixed-mode solution from the stationary state (Fig. 9) and from the oscillatory state (Fig. 10). Our choice of parameters has ensured that the secondary solution is supercritical and thus stable; this can be altered by changing the sign of $b_{1} b_{3}-b_{2} b_{4}$.

The numerical method (PD) will be P-unstable (that is numerically unstable in practice) if either the mode with $k=J-1$ is amplified or the mode with $k=1$ is amplified more rapidly than in (P) itself. Thus the interaction of the two modes is crucial to the approximation of (P) by (PD). This analysis shows a direct mechanism for the exchange and nonlinear generation of energy between the highest and lowest wavenumbers in the discretisation (PD). This possibility, which has important effects 


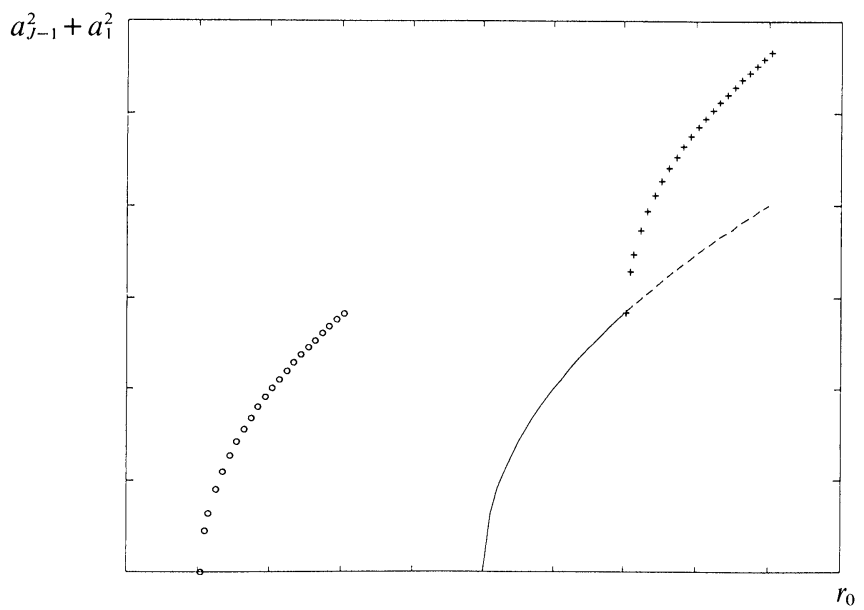

FIG. 9. Bifurcation diagram for (3.3.15), (3.3.16) $\lambda_{0}<0$, $f^{\prime \prime \prime}(0)>0$, and $b_{1} b_{3}-b_{2} b_{4}>0$. Key: - stable stationary solution; -- unstable stationary solution; + stable periodic solution (in $n$ ); unstable periodic solution (in $n$ ).

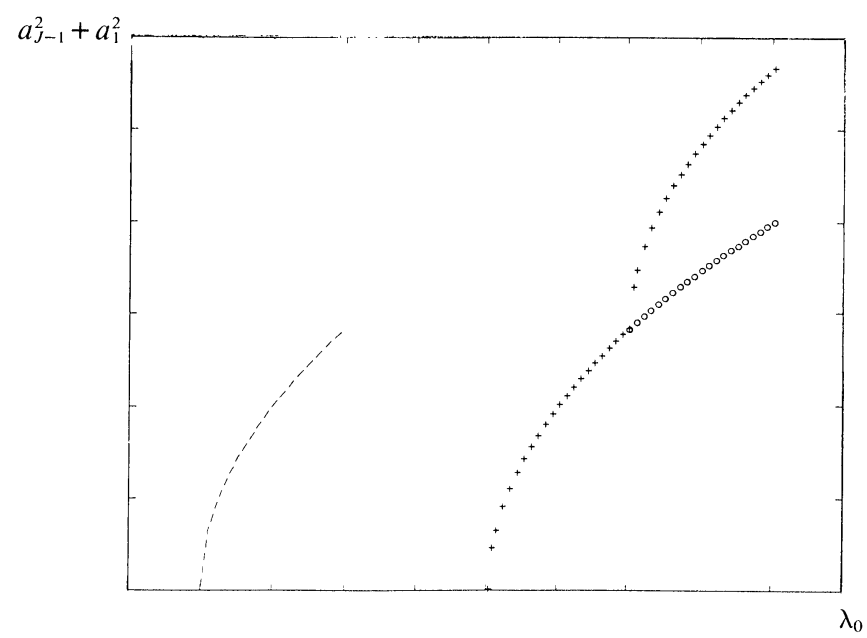

FIG. 10. Bifurcation diagram for (3.3.15), (3.3.16) $r_{0}<0$, $f^{\prime \prime \prime}(0)<0$, and $b_{1} b_{3}-b_{2} b_{4}>0$. See previous figure for key.

on the practical numerical stability of (PD), is the consequence of the interaction between the underlying dynamics of the partial differential equation and the artefacts of the discretisation.

Figure 11 shows the solution of (PD) with $f(u)=\sinh (u)$. The parameters are chosen so that $\Delta x=0.05, \lambda=10.0\left(>\lambda_{c}\right)$, and $r>r_{c}$. The initial data is smooth so that the initial excitation of $a_{J-1}$ is much smaller than that of $a_{1}$. Initially the solution evolves in a stable fashion until the high wavenumber mode $a_{J-1}$ is stimulated by linear effects and through the interaction with $a_{1}$ (see (3.3.15), (3.3.16) and Fig. 11b). For a period of time $a_{J-1}$ swamps the solution as shown in Fig. 11c. However, eventually there is a transfer and generation of energy in the low wavenumber mode $a_{1}$ and the profile in Fig. 11d emerges prior to blowup of the scheme. 


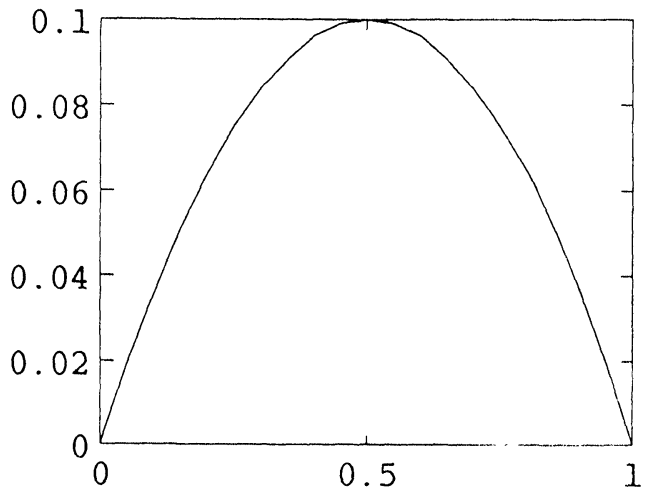

(a)

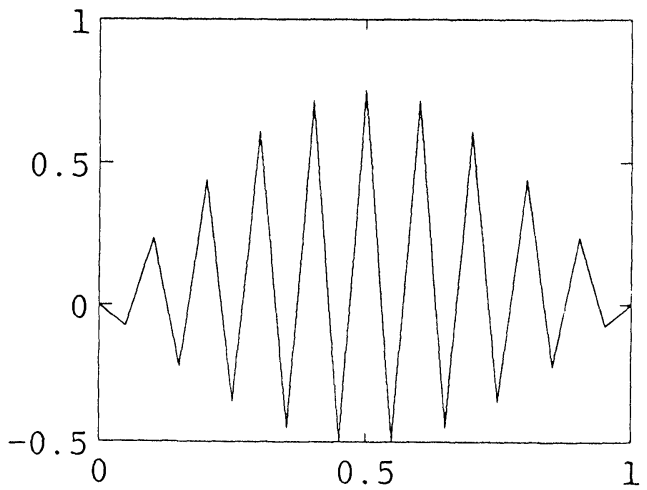

(c)

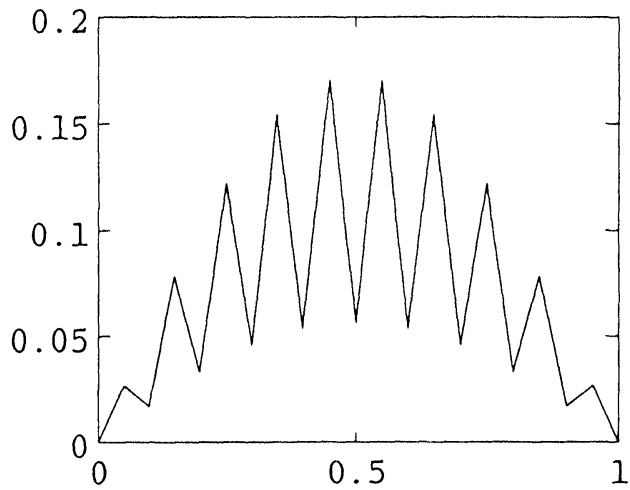

(b)

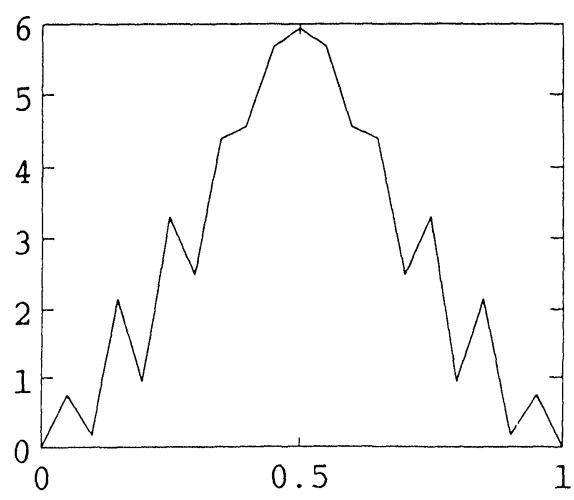

(d)

FIG. 11. Solution of (PD) for $r>r_{c}$ and $\lambda>\lambda_{c}$. (a) $t=0$. (b) $t=500 \Delta t$. (c) $t=625 \Delta t$. (d) $t=760 \Delta t$.

3.4. Bifurcation from infinity. In this subsection we analyse (PD) in case (ii), where $f^{\prime}(0)=0$. It is possible to discuss this problem from the perspective of $\$ 2.4$, namely the bifurcation of steady solutions from $\lambda=\infty$. We choose, however, to consider the case $\theta=\frac{1}{2}$ and discuss the bifurcation of $n$-dependent solutions from $r=\infty$.

When $\theta=\frac{1}{2}$, (PD) reduces to

$$
\begin{gathered}
2\left(u_{j}^{n+1}-u_{j}^{n}\right)=r \delta_{x}^{2}\left(u_{j}^{n+1}+u_{j}^{n}\right)+\lambda \Delta t\left[f\left(u_{j}^{n+1}\right)+f\left(u_{j}^{n}\right)\right], \\
u_{0}^{n+1}=u_{J}^{n+1}=0, \quad u_{j}^{0}=u_{0}(j \Delta x) .
\end{gathered}
$$

Linearising about $u_{j}^{n}=0$ we obtain the solution $v_{j}^{n+1}$, given by

$$
v_{j}^{n+1}=\sum_{k=1}^{J-1} A_{k} \xi_{k}^{n} \sin (k \pi j / J)
$$

where the $A_{k}$ are determined by initial conditions and

$$
\xi_{k}=\frac{1-2 r \sin ^{2}(k \pi / 2 J)}{1+2 r \sin ^{2}(k \pi / 2 J)} .
$$

This expression is obtained from (3.2.3) by setting $\theta=\frac{1}{2}$ and $\lambda=0$, since $\lambda$ actually represents $\lambda f^{\prime}(0)$ in (3.2.3), where $f^{\prime}(0)$ is scaled to be 1 , and here, $f^{\prime}(0)=0$. 
Thus (PD) is numerically stable, according to linear theory, for all values of $r$, since $\left|\xi_{k}\right|<1$. However, as $r \rightarrow \infty$, some of the modes satisfy $\left|\xi_{k}\right| \rightarrow 1$. This suggests that $r=\infty$ is a candidate for a bifurcation point in (3.4.1), (3.4.2).

We may induce $r \rightarrow \infty$ by taking $\Delta x=\Delta t / r_{0}^{1 / 2}$ and letting $\Delta t \rightarrow 0$. In this case

$$
\xi_{k}=\frac{\Delta t-2 r_{0} \sin ^{2}\left(k \pi \Delta t / 2 r_{0}^{1 / 2}\right)}{\Delta t+2 r_{0} \sin ^{2}\left(k \pi \Delta t / 2 r_{0}^{1 / 2}\right)} .
$$

Thus for $k \Delta t \ll 1, \xi_{k} \rightarrow 1$, and for all other $k, \xi_{k} \rightarrow-1$. Since the growth rates approach neutral values, we deduce that $\Delta t=0$ is a candidate for a bifurcation point. As in the continuous case [42], a weakly nonlinear analysis along the lines of the previous section will not be fruitful in this case, since all the modes are near neutral. However, the discussion suggests that some form of $n$-dependent solution bifurcates from $r=\infty$. This, in turn, suggests that some form of finite-amplitude instability is possible, since the solution bifurcating from infinity almost certainly repels data starting in its vicinity; see the discussion of the continuous case in [42].

Of course, in reality, no sensible computations would involve a value $r \gg 1$. However, if the branch of solutions bifurcating from $r=\infty$ extends to moderate values of $r$, it will affect the dynamics of (3.4.1), (3.4.2) considerably, since a finite-amplitude instability can occur.

4. Conclusions. We have examined problems (P) and (PD) in the vicinity of the trivial solution. The important points we have illustrated are:

(i) The growth rates in the linearised problems, $\sigma$ for (P) and $\xi$ for (PD), are necessarily real (see Theorems 2.1.1 and 3.1.1).

(ii) Bifurcation and change in stability are associated with $\operatorname{Re}(\sigma)=0$ for $(\mathrm{P})$ and $|\xi|=1$ for (PD). This is clearly seen by analysing the Laplace and Z-transforms (which are treated in parallel in [50]) for the linearisations of (P) and (PD), respectively.

(iii) The steady bifurcation points at which $\sigma=0$ in (P) correspond to $\xi=1$ in (PD). The positions of the bifurcation points and the amplitudes of the bifurcating solutions are only well approximated by (PD) for $\sqrt{\lambda} / \pi \ll J$, where $J$ is the number of spatial meshpoints (see $\$ 3.2$ ).

(iv) The periodic bifurcation points at which $\xi=-1$ in (PD) have no analogy in (P). They are associated with the onset of numerical instability in (PD) (see §3.2).

(v) The manifestation of numerical instability depends crucially upon the properties of the bifurcating periodic orbit (see $\$ 3.3$, case (a)).

(vi) The presence of spurious steady solutions caused by spatial discretisation can seriously degrade time-dependent calculations (see $\$ 3.2$ and [51]).

(vii) The direct interaction between growing low wavenumber modes present in both (P) and (PD), and high wavenumber modes produced by the discretisation, is fundamental to nonlinear numerical stability (see $\$ 3.3$, case (c)). This result seems to be generic in nonlinear parabolic equations and is demonstrated under entirely different assumptions in [51] (see the discussion in \$3.2).

We make two observations about the relevance of the results obtained here to more general situations. First, our analysis has relied heavily on the simplification of problems (P) and (PD) in the vicinity of the trivial zero solution. However, the secondary bifurcation of numerically destabilising periodic orbits demonstrated in case (c) of $\$ 3.3$ shows that the ideas described hold in the neighbourhood of any steady solution of $(\mathrm{P})$. This assertion is proved in [52], where $(\mathrm{P})$ is generalised to include convection, and the normal form governing the bifurcation is derived. Second, our analysis has also relied on choosing $r \approx r_{c}$, whereas any sensible computation 
would involve $r<r_{c}$. However, it is our objective to describe the manifestations of numerical instability in problems where a dissipative mechanism is balanced by a nonlinearity. For problems more complicated than $(\mathrm{P})$ the linearised stability threshold may not be known explicitly, and thus it is important to be able to recognize the many manifestations of numerical instability in nonlinear problems. The analysis of the simple problems (P) and (PD) enables us to achieve this.

Finally, we conclude by stating our belief that the analysis of numerical methods will benefit increasingly from an interaction with bifurcation theory and dynamical systems theory. It is important to study the bifurcation diagram generated by a numerical approximation and compare it with that of the underlying continuous problem. In practice this means examining the numerical method for all ranges of the physical parameters at once rather than looking at convergence for a fixed set of physical parameters. This reveals critical scalings of the natural physical parameters of the problem in terms of the discretisation parameters. In the long term, analysis should aim toward proving convergence to a given bifurcation diagram rather than to a solution for a fixed parameter set. Similarly, it is valuable to consider the numerical method as a dynamical system and to compare the flow generated by the numerical method with that generated by the underlying continuous problem. Such analysis reveals critical scalings of the initial data in terms of the discretisation parameters. In contrast, classical analysis focuses on convergence for fixed initial conditions. Recent rigourous analysis of numerical methods for ordinary differential equations considered as dynamical systems has been achieved by (among others) Beyn [3] and Iserles [24].

Acknowledgments. I am grateful to Professors L. N. Trefethen and J. M. SanzSerna for a number of comments and suggestions which improved earlier versions of this paper. The work presented here is based in part on a seminar given at the Numerical Analysis Group, Oxford University in 1986.

\section{REFERENCES}

[1] L. BAuer, H. B. Keller, AND E. L. Reiss, Multiple eigenvalues lead to secondary bifurcation, SIAM Rev., 17 (1975), pp. 101-122.

[2] T. B. Benjamin and J. E. Feir, The disintegration of wave trains on deep water. Part 1. Theory, J. Fluid Mech., 27 (1967), pp. 417-430.

[3] W.-J. BEYN, On the numerical approximation of phase portraits near stationary points, SIAM J. Numer. Anal., 24 (1987), pp. 1095-1113.

[4] E. BoHL, Discrete versus continuous models for dissipative systems, in Numerical Methods for Bifurcation Problems, T. Kupper, H. D. Mittelmann, and H. Weber, eds., Birkhäuser-Verlag, Boston, 1984.

[5] F. Brezzi, S. Ushikı, AND H. FujII, Real and ghost bifurcation dynamics in difference schemes for ordinary differential equations, in Numerical Methods for Bifurcation Problems, T. Kupper, H. D. Mittelmann, and H. Weber, eds., Birkhäuser-Verlag, Boston, 1984.

[6] W. L. BriggS, A. C. Newell, AND T. SARIE, Focusing: a mechanism for instability of nonlinear finitedifference equations, J. Comp. Phys., 51 (1983), pp. 83-106.

[7] N. ChafEe AND E. Infante, A bifurcation problem for a nonlinear parabolic equation, Appl. Anal., 4 (1974), pp. 17-37.

[8] S.-N. Chow and J. Hale, Methods of Bifurcation Theory, Springer-Verlag, Berlin, New York, 1982.

[9] A. Cloot AND B. M. HeRBSt, Grid resonances, focusing and Benjamin-Feir instabilities in leapfrog time discretisation, J. Comp. Phys., 75 (1988), pp. 31-53.

[10] A. A. Craik, Wave interactions and fluid flows, Cambridge University Press, Cambridge, U.K., 1985.

[11] J. DE Frutos AND J. M. SAnz-SERna, h-dependent stability thresholds avoid the need for a priori bounds in nonlinear convergence proofs, in Computational Mathematics III, S. O. Fatunla, ed., Boole Press, Dublin, 1988. 
[12] P. G. Drazin AND W. H. Reid, Hydrodynamic Stability Theory, Cambridge University Press, Cambridge, U.K., 1981.

[13] J. EASTWOOD AND W. ARTER, Spurious behaviour of numerically computed fluid flow, iMA J. Numer. Anal., 7 (1987), pp. 205-222.

[14] P. C. Fife, Mathematical Aspects of Reacting and Diffusing Systems, Lecture Notes in Biomath. 28, Springer-Verlag, Berlin, New York, 1978.

[15] — Semilinear elliptic boundary value problems with small parameters, Arch. Rational Mech. Anal., 52 (1973), pp. 205-232.

[16] B. ForNBERG, On the instability of Leap-Frog and Crank-Nicolson approximations of a nonlinear partial differential equation, Math. Comp., 27 (1973), pp. 45-57.

[17] M. A. Golubitsky And D. G. Schaeffer, Singularities and Groups in Bifurcation Theory, Vol. I, Appl. Math. Sci., 51 (1985).

[18] D. F. Griffiths AND A. R. Mitchell, Stable periodic bifurcations of an explicit discretization of a nonlinear partial differential equation in reaction diffusion, IMA J. Numer. Anal., 8 (1988), pp. 435-454.

[19] J. Guckenheimer and P. Holmes, Nonlinear Oscillations, Dynamical Systems and Bifurcation of Vector Fields, Appl. Math. Sci., 42 (1983).

[20] P. Hartman, Ordinary Differential Equations, John Wiley, New York, 1981.

[21] D. Henry, The Geometric Theory of Semilinear Parabolic Equations, Lecture Notes in Math. 840, Springer-Verlag, Berlin, New York, 1981.

[22] B. M. HerbSt AND A. ClOOT, The modulational instability of the Korteweg-deVries equation, Tech. Report 1/88, Dept. of Applied Mathematics, University of the Orange Free State, Bloemfontein, South Africa, 1988.

[23] D. HoFF, Stability and convergence of finite difference methods for systems of nonlinear reaction diffusion equations, SIAM J. Numer. Anal., 15 (1978), pp. 1161-1171.

[24] A. ISERLES, Stability and dynamics for nonlinear ordinary differential equations, Report DAMTP 1988/NA1, University of Cambridge, Cambridge, U.K., 1988.

[25] A. K. Kapila, Asymptotic Treatment of Chemically Reacting Systems, Pitman, Boston, London, 1982.

[26] J. P. KEENER, Secondary bifurcation in nonlinear diffusion reaction equations, Stud. Appl. Math., 55 (1977), pp. 187-211.

[27] H. KIELHOFER, On the Liapunov stability of stationary solutions of semilinear parabolic equations, J. Differential Equations, 22 (1976), pp. 193-208.

[28] D. L. Kreider, R. G. Kuller, D. R. Ostberg, and F. W. Perkins, Introduction to Linear Analysis, John Wiley, New York, 1966.

[29] T. Kupper, H. D. Mittelmann, and H. Weber, Numerical Methods for Bifurcation Problems, Birkhäuser-Verlag, Boston, 1984.

[30] J. C. Lopez-Marcos AND J. M. Sanz-Serna, Stability and convergence in numerical analysis II: The definition of stability in nonlinear problems, unpublished manuscript. Condensed version in Numerical Treatment of Differential Equations, Proc. of the Fourth Seminar NUMDIFF-4, Halle, Federal Republic of Germany, 1987, K. Strehmel, ed., Teubner-Texte Math. 104, Leipzig, Federal Republic of Germany, 1988.

[31] - Stability and convergence in numerical analysis III: Linear investigation of nonlinear stability, IMA J. Numer. Anal. 8 (1988), pp. 71-84.

[32] D. Ludwig, D. G. Aronson, And H. F. Weinberger, Spatial patterning of the spruce budworm, J. Math. Biol. 8 (1979), pp. 217-225.

[33] J. Marsden and M. MCCracken, The Hopf Bifurcation and its Applications, Appl. Math. Sci., 19 (1976).

[34] H. Matano, Asymptotic behaviour and stability of solutions of semilinear diffusion equations, Publ. RIMS, Kyoto Univ., 15 (1979), pp. 401-454.

[35] B. J. MаткоwSкy, A simple nonlinear dynamic stability problem, Bull. Amer. Math. Soc., 76 (1970), pp. 620-625.

[36] A. R. Mitchell AND D. F. Griffiths, Beyond the linearised stability limit in nonlinear problems, in Numerical Analysis, D. F. Griffiths and G. A. Watson, eds., Research Notes in Math. 140, Pitman, Boston, 1986.

[37] D. W. MoOre, Resonances introduced by discretization, IMA J. Appl. Math., 31 (1983), pp. 1-11.

[38] A. C. Newell, Finite amplitude instabilities of partial difference equations, SIAM J. Appl. Math., 33 (1977), pp. 133-160.

[39] J. NORBURY, Interior transition layers for semilinear elliptic problems are located near extremal geodesics, in Free Boundary Problems: Applications and Theory. Vol. IV, A. Bossavit, A. Damlamian, and M. Fremond, eds., Res. Notes in Math. 121, Pitman, Boston, 1985. 
[40] N. A. Phillips, An example of nonlinear computational instability, in The Atmosphere and the Sea in Motion, B. Bolin, ed., Rockefeller Institute Press, New York, 1959.

[41] R. D. Richtmyer And K. W. Morton, Difference Methods for Initial Value Problems, John Wiley, New York, 1967.

[42] S. Rosenblat and S. H. Davis, Bifurcation from infinity, SIAM J. Appl. Math., 37 (1979), pp. 1-19.

[43] J. M. SAnz-Serna, Studies in numerical nonlinear stability III: augmented Hamiltonian systems, SIAM J. Appl. Math., 47 (1987), pp. 92-108.

[44] — Runge-Kutta schemes for Hamiltonian systems, BIT, 28 (1988), pp. 877-883.

[45] S. W. SchoоmвiE, A discrete multiple scales analysis of a discrete version of the Korteweg-deVries equation, Report NA/109, University of Dundee, Dundee, U.K., 1987.

[46] R. Shreiber And H. B. Keller, Spurious solutions of driven cavity calculations, J. Comp. Phys. 49 (1983), pp. 165-172.

[47] S. Sivaloganathan, Ph.D. thesis, Numerical Analysis Group, Oxford University, 1986.

[48] D. M. Sloan and A. R. Mitchell, On nonlinear instabilities in Leap-Frog finite difference schemes, J. Comp. Phys., 67 (1986), pp. 372-395.

[49] J. Smoller, Shock Waves and Reaction Diffusion Equations, Springer-Verlag, New York, 1983.

[50] G. STrang, Introduction to Applied Mathematics, Wellesley-Cambridge Press, Wellesley, MA, Cambridge, MA, 1986.

[51] A. M. StUART, A note on high/low wave number interactions in spatially discrete parabolic equations, IMA J. Appl. Math., 1989, to appear.

[52] — Linear instability implies spurious periodic solutions, submitted to IMA J. Numer. Anal., 1988.

[53] J. T. StUART, On the nonlinear mechanics of wave disturbances in stable and unstable parallel flows. Part 1: The basic behaviour in plane Poiseuille flow, J. Fluid Mech., 9 (1960), pp. 353-370.

[54] L. N. Trefethen, Group velocity in finite difference schemes, SIAM Rev., 24 (1982), pp. 113-135.

[55] - Dispersion, dissipation and stability, in Numerical Analysis, D. F. Griffiths and G. A. Watson, eds., Res. Notes in Math. 140, Pitman, Boston, 1986.

[56] J. WATSON, On the nonlinear mechanics of wave disturbances in stable and unstable parallel flows. Part 2: The development of solutions for plane Poiseuille flow and for plane Couette flow, J. Fluid Mech., 9 (1960), pp. 371-389.

[57] J. A. C. Weideman and L. N. Trefethen, The eigenvalues of second-order spectral differentiation matrices, SIAM J. Numer. Anal., 25 (1988), pp. 1279-1298.

[58] R. WeISs, Bifurcation in difference approximations to two-point boundary value problems, Math. Comp., 29 (1975), pp. 746-760. 